\title{
基于离子型忆阻器的神经形态系统: 从材料、器件到芯片
}

\author{
温娟, 黄鹤鸣, 王哲, 郭新* \\ 华中科技大学材料科学与工程学院, 材料成形与模具技术国家重点实验室, 武汉 430074 \\ *联系人, E-mail: xguo@hust.edu.cn \\ 2021-10-15 收稿, 2021-12-03 修回, 2021-12-28 接受, 2022-01-11 网络版发表 \\ 国家重点研发计划(2018YFE0203802)和华为技术合作项目(YBN2019055139)资助
}

\begin{abstract}
摘要 现有的“冯·诺依曼”体系因其计算与存储分离的特点难以满足人工智能、物联网等新技术在能耗和速率方 面的发展需求, 而存算一体化的类脑计算方案有望突破这一瓶颈. 忆阻器是一种新型微电子基础器件, 其电阻可通 过外界电场调节, 而且忆阻器在结构与功能上接近于生物大脑中的突触和神经元, 利用其构建的忆阻神经网络具 有小尺寸、高速和低能耗等优良特性, 有助于实现更接近生物大脑的人工智能. 本文对忆阻器领域近年来的研究 进展进行了全面的综述: 在材料层面, 根据阻变机理介绍了忆阻器的不同类型; 在器件层面, 结合当前忆阻器的应 用背景论述了基于离子型忆阻器的性能要求及优化措施; 在系统层面, 讨论了离子型忆阻器作为忆阻突触、忆阻 神经元的应用, 并进一步概述了忆阻神经网络的网络结构、学习算法和硬件实现, 最后对基于离子型忆阻器的神 经形态芯片所面临的挑战和未来前景进行了总结与展望.
\end{abstract}

关键词忆阻器, 忆阻突触, 忆阻神经元, 忆阻神经网络, 神经形态芯片

自1956年诞生以来, 人工智能飞速发展, 已在自然 语言处理、专家系统、识别系统、医学等诸多智能领 域展现出惊人的能力, 而这种进步背后的硬件基础是 芯片算力的提升. 伴随着数据量的增大、算法的革新 和算力的提升, 人类社会正从信息化迈向智能化, 其中 人工智能芯片是智能化社会的基石和战略制高点. 广 义上讲, 只要能够运行人工智能算法的芯片都可叫做 人工智能芯片. 但是目前, 芯片的操作频率和晶体管的 密度已经发展到了瓶颈期, 这主要源于芯片行业正面 临的两大困境: 一是摩尔定律的“失效”, 随着电路的集 成密度增大, 晶体管数量增加, 器件发热问题也愈发严 重, 发热量必会达到难以逾越的阈值; 另一个是现行计 算机架构的冯·诺依曼“内存墙”, 即运算单元与存储单 元相互分离 ${ }^{[1]}$, 数据在运算单元与存储单元间的频繁 传输, 导致能耗过大. 有研究指出, 对于许多计算任务,
例如运动物体、图像和语音识别等, 大多数的能量和 时间消耗在数据移动中而不是计算上 ${ }^{[2]}$. 为了解决或 者避免以上问题, 继续推动人工智能的发展, 现代技术 从器件到系统架构设计等多个角度来克服这些障碍. 其中，一类是面向当前的深度学习技术开发的人工神 经网络(artificial neural network, ANN)芯片, 在ANN芯 片中, 信息以数字比特、时钟频率和高低电平来表示; 另一类是设计类似于人脑工作的计算架构, 即脉冲神 经网络(spiking neural network, SNN), 在SNN芯片 中, 信息被编码为电脉冲序列, 通过在相对低频下模 仿真正的生物神经元和突触的行为来实现超低能耗 计算.

近年来, 新兴的非易失性存储器被引人现行的内 存层次结构以突破冯·诺依曼瓶颈 ${ }^{[3]}$, 这便是基于非易 失存储器的存内计算(in-memory computing). 存内计算

引用格式: 温娟, 黄鹤鸣, 王哲, 等. 基于离子型忆阻器的神经形态系统: 从材料、器件到芯片. 科学通报, 2022, 67: 1054-1071 Wen J, Huang H M, Wang Z, et al. Neuromorphic systems based on ionic memristors: From materials, devices to chips (in Chinese). Chin Sci Bull, 2022, 67: 1054-1071, doi: 10.1360/TB-2021-1072 
通过存算一体的系统来消除耗能耗时的数据搬运，这 种方法类似于人脑中处理信息的计算方案：在人脑中, 信息在神经元和突触组成的稀疏网络中进行处理，计 算与存储一体 ${ }^{[4]}$. 这种新架构需要器件同时进行存储 与计算，而该过程通常是通过器件的物理性质或其他 物理定律来完成的, 例如, 欧姆定律和电路中的基尔霍 夫定律. 这些新式存储器都有独特的存储原理, 包括电 阻式随机存储器(resistive random access memory, RRAM) ${ }^{[5]}$ 、相变存储器(phase change memory, $P C M)^{[6]}$ 、磁阻式存储器(magneto-resistive random access memory, MRAM) ${ }^{[7]}$ 和铁电存储器(ferroelectric random access memory, FeRAM) ${ }^{[8]}$. 其中, RRAM具有高密 度、快速和随机访问的特点, 并且具备丰富的离子动 力学，可以大大简化内存层次结构，提高系统性能. 事 实上, 2008年, 惠普实验室在寻找新型存储器时发现, 阻变器件的性质与预言的第4种电路元件一一忆阻器 有诸多相似之处, 因此称其为忆阻器 ${ }^{[9]}$.

基于忆阻器的人工智能芯片，也称为神经形态芯 片, 是克服当今计算架构面临障碍的一个重要途径, 并 且与当前和未来的计算需求极为相关, 将在认知处 理、大数据分析和基于低能耗智能系统的物联网中发 挥重大作用. 本文首先根据阻变机理对主要的忆阻器 类型进行简单介绍，结合当前忆阻器的应用背景论述 忆阻器的性能要求, 并概括近些年研究人员为满足这 些性能要求, 对器件性能进行优化的研究进展. 然后, 概述忆阻器作为忆阻突触和忆阻神经元的要求与应用, 基于此, 进一步介绍忆阻神经网络的网络结构、学习 算法和硬件实现。

\section{1 忆阻器}

忆阻器是一种具有记忆效应的电路器件, 通常具 有金属/绝缘体/金属的“三明治”结构，主要依靠内部离 子的迁移来改变器件的电学性质, 在外加电场(直流或 脉冲信号)的作用下，器件能在高低电导态之间发生可 逆转变. 忆阻器具有结构简单、操作速度快、能耗 低、成本低、高密度集成和性能丰富等特点. 亚琛工 业大学Waser ${ }^{[10]}$ 根据主导阻变的物理化学现象, 大致将 阻变机理分为电化学金属化效应(electrical metallization effect, ECM)、价态变化效应(valence change memory effect, VCM)、热化学效应(thermochemical memory effect, TCM)、纯电子效应(electrostatic/electronic effects) ${ }^{[11 \sim 13]}$ 和相变记忆效应(phase change memory effect,
$\mathrm{PCM})^{[14,15]}$. 本文主要介绍较为常见的 $\mathrm{ECM}$ 和 VCM 效应.

\section{1 忆阻器的阻变机理}

\subsubsection{ECM}

以ECM为导电机理的忆阻器由 1 个可氧化的顶电 极 (阳极, 如 $\mathrm{Ag}$ 和 $\mathrm{Cu}$ )、1个相对惰性的底电极(阴极, 如 $\mathrm{W}$ 和 $\mathrm{Pt}$ )，以及 2 个电极之间夹着的金属氧化物层(如 $\mathrm{SiO}_{2}{ }^{[16]} 、 \mathrm{Al}_{2} \mathrm{O}_{3}{ }^{[17]}$ )组成. 在阻变过程中, 器件中的阻变 层会形成一个金属导电通道. 如图1(a)所示，活性电极 $\mathrm{M}$ 在氧化反应过程中形成金属阳离子 $\mathrm{M}^{n+}: \mathrm{M} \rightarrow \mathrm{M}^{n+}+$ $n \mathrm{e}^{-}$, 在外加电场作用下, $\mathrm{M}^{n+}$ 在电介质层中向惰性电极 一侧迁移; 在惰性电极侧, $\mathrm{M}^{n+}$ 得到电子发生还原反应: $\mathrm{M}^{n+}+n \mathrm{e}^{-} \rightarrow \mathrm{M}$ ，转变成金属 $\mathrm{M}$, 并最终形成金属导电细 丝, 器件转变为低阻态(low resistance state, LRS), 这个 过程被称为 SET; 当施加电压的极性相反时, 导电细丝 可被熔解, 器件又回到高阻态(high resistance state, $\mathrm{HRS}$ ), 这个过程被称为 RESET ${ }^{[18]}$. Guo等人 ${ }^{[19]}$ 创新性 地使用水作为电介质制备了 $\mathrm{Pt} / \mathrm{H}_{2} \mathrm{O} / \mathrm{Ag}$ 器件, 直观地观 察到了细丝从Pt电极指向 $\mathrm{Ag}$ 电极的形成过程. Celano 等人 ${ }^{[20]}$ 制备了 $\mathrm{Cu} / \mathrm{Al}_{2} \mathrm{O}_{3} / \mathrm{TiN}$ 忆阻器，其 $I-V$ 扫描曲线如 图1(b)所示，利用3D层析成像技术观察了导电细丝形 成过程. Choi等人 ${ }^{[21]}$ 利用外延生长在硅上的单晶 $\mathrm{SiGe}$ 层作为阻变层制备了 $\mathrm{ECM}$ 器件, 利用 $\mathrm{SiGe}$ 层中的位错 将金属导电细丝限制在一个确定的一维通道中，极大 地提高了器件的开关比、均一性、保持特性和耐久性.

\subsubsection{VCM}

以VCM为导电机理的忆阻器主要由非活性金属电 极/氧化物/惰性电极组成, 器件中起主导因素的大多是 氧离子或者氧空位, 为简化导电模型, 通常采用氧空位 来描述导电过程. 如图1(c)所示, 在电场作用下, 氧空位 发生迁移, 导致阻变层材料的组成不均匀, 金属元素发 生价态变化. 同时, 氧空位的排布会形成非金属导电细 丝, 使器件表现出导电细丝开关行为. 另外, 当电极与 阻变层之间存在肖特基势垒时，氧空位在界面处的积 累会导致势垒高度的变化, 进而影响阻变开关过程. 因 此, 基于VCM的器件, 其导电机理往往是导电细丝和界 面势垒的共同作用 ${ }^{[22]}$. 该器件的重要特征在于阻变层 本身参与氧化还原反应，因此其一般具有更稳定的阻 变性能和更好的疲劳性能(endurance). 以 $\mathrm{Ta} / \mathrm{TaO}_{x} / \mathrm{Pt} / \mathrm{Ti}$ 器件为例 ${ }^{[23]}$, 如图1(d)所示, 施加正向扫描电压时, 氧 空位在电场作用下向阴极迁移，在阴极获得电子并累 
(a) HRS

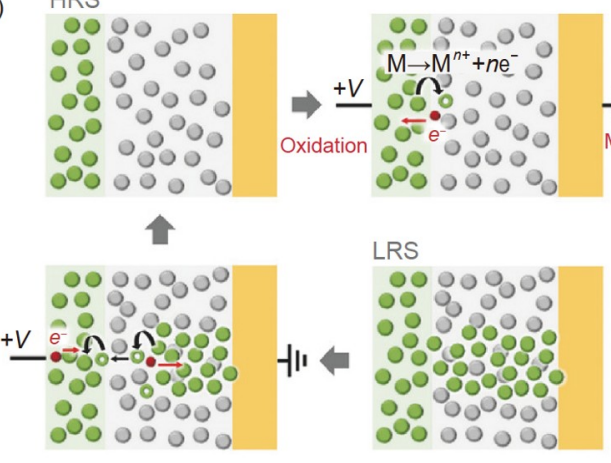

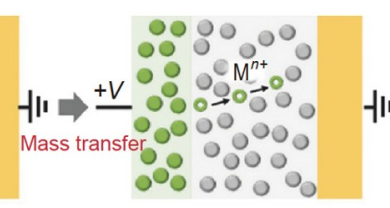

Reduction

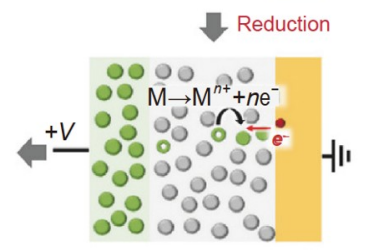

(c) HRS

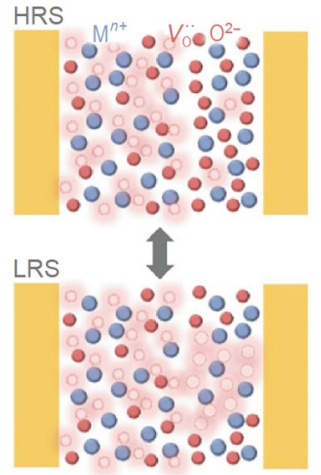

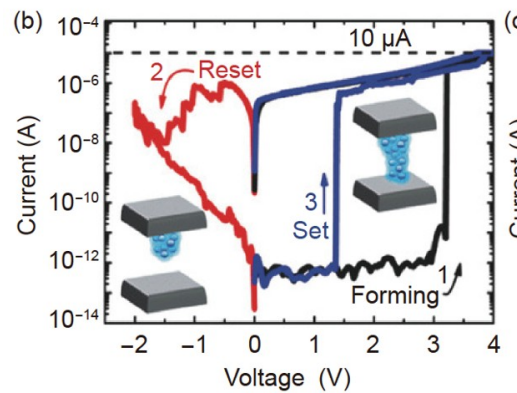
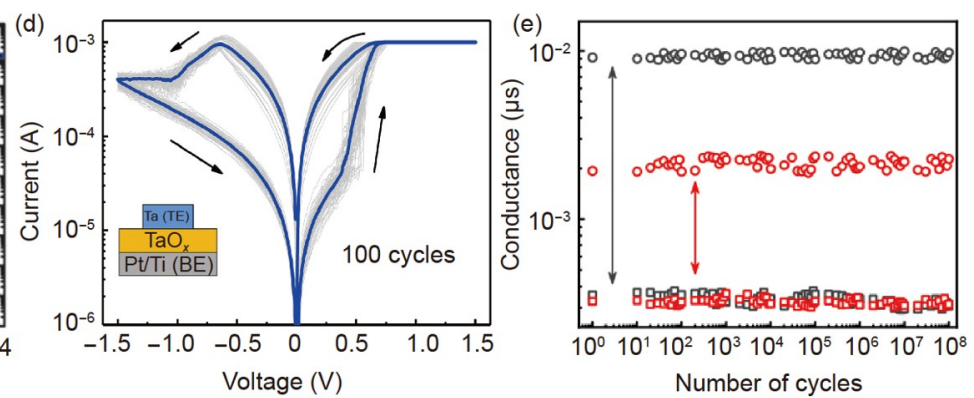

图 1 器件阻变机理与性能. (a) $\mathrm{ECM}$ 器件阻变过程示意图 ${ }^{[18]}$; (b) $\mathrm{Cu} / \mathrm{Al}_{2} \mathrm{O}_{3} / \mathrm{TiN}^{\mathrm{g}}$ 件的 $I-V$ 曲线 ${ }^{[18]}$; (c) $\mathrm{VCM}$ 器件阻变过程示意图 ${ }^{[18]}$; (d) $\mathrm{Ta} / \mathrm{TaO}_{x} /$ $\mathrm{Pt} / \mathrm{Ti}$ 器件的 $I-V$ 曲线 ${ }^{[23]}$; (e) 疲劳特性曲线 ${ }^{[24]}$

Figure 1 Resistive switching mechanisms and performances of devices. (a) Schematic illustration of the resistive switching process in the ECM device ${ }^{[18]}$; (b) hysteretic $I-V$ curves of the $\mathrm{Cu} / \mathrm{Al}_{2} \mathrm{O}_{3} / \mathrm{TiN}_{\text {device }}{ }^{[18]}$; (c) schematic illustration of the resistive switching process in the VCM device ${ }^{[18]}$; (d) $I-V$ curve of the $\mathrm{Ta} / \mathrm{TaO}_{x} / \mathrm{Pt} / \mathrm{Ti}$ device ${ }^{[23]}$; (e) endurance measurement ${ }^{[24]}$

积, 最终形成氧空位导电细丝, 器件从HRS转变为LRS, 完成SET过程; 施加负向扫描电压时，导电细丝产生大 量焦耳热, 细丝周围的温度急剧上升, 导致导电细丝熔 解并断裂, 器件从LRS转变为HRS, 完成RESET过程. 清 华大学吴华强课题组 ${ }^{[24]}$ 在 $\mathrm{SrTiO}_{3}$ 基底上生长了铁铝酸 钙矿石 $(\mathrm{BM})-\mathrm{SrTiO}_{3} / \mathrm{SrRuO}_{3}$ 外延薄膜, 采用 $\mathrm{Au}$ 作为顶 电极制备了 $\mathrm{VCM}$ 器件. 图1(e)是器件在 $10^{8}$ 次循环下的 HRS和LRS, 展现了出色的疲劳性能.

目前, 多种氧化物被报道具有阻变效应, 包括二元 氧化物(如 $\mathrm{TiO}_{x}{ }^{[25]} 、 \mathrm{TaO}_{x}{ }^{[26-29]} 、 \mathrm{WO}_{x}{ }^{[30]} 、 \mathrm{HfO}_{x}^{[31]}$ 等)、 复杂氧化物 ${ }^{[32]}$ (如 $\mathrm{SrTiO}_{3}{ }^{[33 ~ 35]} 、 \mathrm{La}_{0.7} \mathrm{Ca}_{0.3} \mathrm{MnO}_{3}{ }^{[36,37]}$ 等)、氮化物 ${ }^{[38]}$ 和有机材料等 ${ }^{[39 ~ 41]}$. 虽然很多氧化物都 能制备成忆阻器, 但选择不同材料, 器件所获得性能大 不相同, 故为获得期望的优异性能, 必须先从材料选择 人手. 器件在阻变过程中会出现一个绝缘相和一个导 电相，工作过程中会不可避免地产生大量的焦耳热 ${ }^{[42]}$, 引起高温. 在此过程中, 两个相没有发生化学反应形成 新的相是获得可靠阻变的基础. 简单的材料体系中有 两个热力学稳定的固态相, 意味着它们即使在高温下
也不会相互反应形成新相. 例如, $\mathrm{MeO}_{x}$ 相是绝缘相, $\mathrm{Me}$ 相则相对导电, 充当导电通道; 作为通道材料的 $\mathrm{Me}$ 相应具有大的氧溶解度, 使其可以轻松容纳可移动物 质的进出，而不会因多次循环工作而丢失这些物质. Ta-O体系和Hf-O体系均符合以上标准，是制备可靠忆 阻器的优选材料.

\section{2 忆阻器的性能要求}

忆阻器作为构建人工突触和人工神经元的基础元 件, 不同的应用场景对性能有不同的要求. 相较于阻变 类型，在人工突触或人工神经元的应用过程中更关注 忆阻器的保持特性(retention), 即阻变后电导随时间的 变化关系. 若电导随着时间发生变化并最终能够回到 初始态, 则该器件为易失性(volatile) 器件; 若电导随着 时间变化但无法回到初始态, 则该器件为部分易失 (partially volatile) 器件; 若电导随着时间变化保持不变, 则该器件为非易失(non-volatile)器件. 人工神经元需要 具有易失性的器件 ${ }^{[43]}$, 或者具有易失性阈值转变 (threshold switching, TS)的忆阻器 ${ }^{[44]}$ 来完成对时空信 
息的整合，这类器件对稳定性、可靠性和均一性要求 较高. 在ECM和VCM两类忆阻器中都有不同的易失类 型，但导电机理为ECM的忆阻器中的活性金属扩散更 快, 更容易出现易失性阈值转变的特性, 适合用于神经 元功能的模拟; 而导电机理为VCM的忆阻器更有利于 实现非易失的模拟(analog)特性，更适合用于突触功能 的模拟. 非易失性器件作为实现算力提升和功能构建 的关键, 有着迫切的规模化需求, 对性能指标也要求较 多，如良好的权值更新线性、对称性等是神经形态芯 片能否具有竞争力的关键.

对于神经形态芯片，人们往往从计算密度、能量 效率、计算精度和学习能力 4 个基准指标来评估其竞 争力: (1) 计算密度, 即芯片的面积效率, 是设计大规模 神经形态芯片的关键指标, 这就要求突触器件具有较 好的集成能力. (2) 能耗是基于硬件的神经网络系统和 生物大脑之间差距的关键指标. 在训练阶段, 大部分能 量消耗在突触器件的权重更新操作上; 而在推理阶段, 大部分能量消耗在外围电路, 通常是模数转换器(ana- log-digital converter, ADC)或数模转换器(digital-analog converter, DAC) 和突触器件的读操作上. 随着架构的优 化和计算密度的提高, 突触器件读操作消耗的能量将 成为主导, 因此, 每次操作的能耗是评价神经形态芯片 的另一个关键指标. (3) 高计算精度在芯片的整个生命 周期中都是必要的，神经形态芯片的计算精度将受到 器件非理想因素和电路噪声的影响, 例如热噪声和可 靠性等问题. (4) 未来越来越多的边缘计算应用场景对 学习能力提出越来越高的要求. 如需提高芯片的计算 密度、能耗效率和计算精度, 忆阻器的性能, 如电导态 数量、电导态的稳定性、均一性和电导态更新的线性 等需进一步优化.

图2(a)显示了不同应用条件对器件性能的要求, 其中给定轴上更大的值表示对相应指标的要求更高, 图2(b) (h) 给出了器件的性能示意图. 其中, 图2(b)为电 导态数量, 器件的电导值表示神经网络中的权值, 决定 了权值调节精度, 如果电导态数量不够多, 神经网络中 的许多权值只能共享同一数值，使其拟合能力大大降

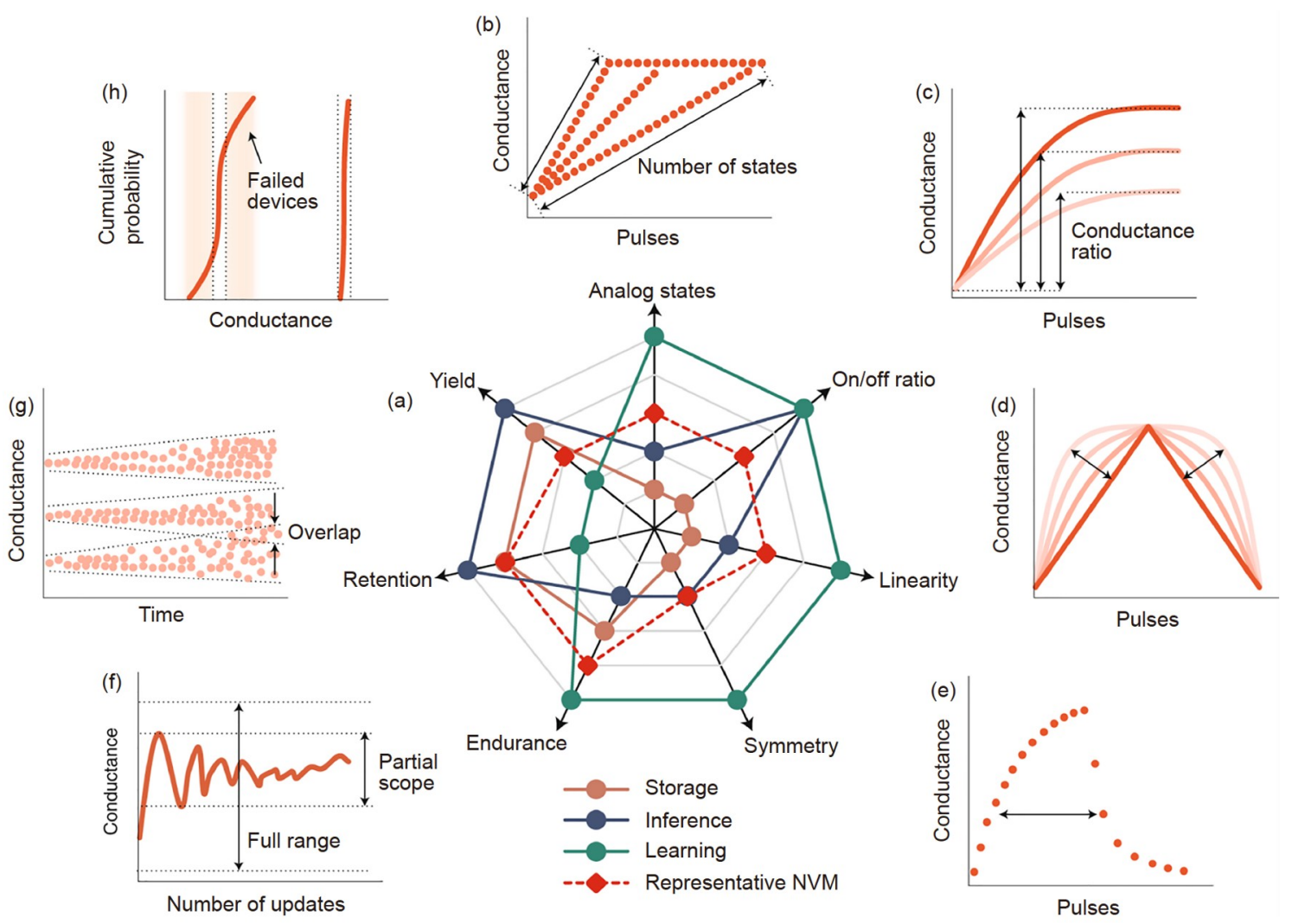

图 2 器件的性能指标要求 ${ }^{[45]}$. (a) 不同应用条件对器件性能的要求; (b) 电导态数量; (c) 开/关比; (d) 电导态更新线性; (e) 更新对称性; (f) 瘦劳 性能; (g) 保持特性; (h) 良率

Figure 2 Application-dependent device metric requirements ${ }^{[45]}$. (a) Ranking of the device requirements for prospective applications; (b) number of conductance states; (c) on/off ratio; (d) updating linearity; (e) symmetry; (f) endurance; (g) retention; (h) yield 
低, 影响计算精度. 开/关比是指最大电导值 $G_{\text {max }}$ 与最小 电导值 $G_{\text {min }}$ 的比值(图2(c)), 也称为动力学范围, 决定了 将算法中的权值映射到器件电导的能力. 如果动力学 范围不够大, 无论电导态数量多大, 其映射的权值范围 都会过小, 从而影响计算精度. 电导态更新线性(图2(d)) 是指在连续施加增强性脉冲和抑制性脉冲过程中, 电 导值随脉冲数量变化曲线的线性; 增强过程与抑制过 程中电导值变化曲线的对称性称为电导更新对称性 (图2(e)), 线性和对称的电导态更新在网络训练阶段至 关重要. 疲劳性能(图2(f))是指器件的使用寿命, 由于 片上学习过程中需要反复进行权重调节, 故对器件疲 劳性能要求很高. 保持特性(图 $2(\mathrm{~g})$ ) 是指电导随时间的 变化关系, 在推理过程中要求每个器件保持稳定的电 导值, 电导的任何随机变化都可能导致计算精度下降. 良率(图2(h))是指器件的成品率, 在推理过程中对其要 求较高. 综上所述, 在训练阶段时, 器件对 analog特 性、开关比、疲劳特性、电导态更新线性和对称性要 求更高; 在推理阶段, 器件对保持性能和良率要求更高, 因此, 可根据器件不同的应用场景选择合适的器件 性能.

\section{2 忆阻突触}

在神经科学中, 神经网络的基础单元是突触和神 经元. 突触是连接突触前神经元和突触后神经元的节 点, 每个节点具有一定的权值, 突触根据权值的大小确 定两个神经元之间的信号传输效率. 突触权值也可以 根据突触前/后神经元的活动进行调节, 这也被称为突 触可塑性(synaptic plasticity), 是大脑学习的基础 ${ }^{[46]}$. 忆 阻器在结构和功能特性上都表现出与生物突触的相似 性: 在结构上, 忆阻器是典型的两端子器件, 而突触和 相应的前后神经元也同样形成两端子结构; 在功能上, 忆阻器件的输出电流由欧姆定律决定: $I=g V$, 其中, 电 压 $V$ 代表突触前神经元的信号，电流 $I$ 代表突触后神经 元的响应, 而忆阻器的电导 $g$ 可以代表突触权值. 在持 续的外部电压或电流信号激励下, 器件电导表现出连 续增加或降低, 这类似于突触权值的增强或减弱 ${ }^{[47]}$. 因 此, 忆阻器被认为是人工突触器件的有力之选.

\section{1 忆阻突触的非理想特性优化}

忆阻突触即基于忆阻器的人工突触, 要求器件具 有良好的权值更新线性、对称性、较好的保持特性和 均一性, 但是在实际测试过程中, 忆阻突触存在着各种 非理想特性, 这些非理想特性会影响后续忆阻神经网 络的性能. 因此, 研究人员在非理想特性的优化上作了 很多探索. 如表1所示, 采取的措施主要有3种: 导电细 丝宽度调节、界面势垒调节和导电通道成分调节. 这3 种方式皆可作用于VCM器件, 而ECM器件主要采用导 电细丝宽度调节和导电通道成分调节两种方式. 例如, 在器件中增加阻挡层限制离子扩散或插人热增强层 (thermal enhanced layer, TEL)、电热调制层(electrothermal modulation layer, ETML)提高器件的工作温度, 有利于获得更好的电导更新线性. 通过控制离子的扩 散能力来实现保持特性的调控, 如当离子在氧化物中 的扩散能力下降时, 自扩散现象减弱, 器件的非易失性

\section{表 1 忆阻器非理想特性的优化}

Table 1 Optimization of nonideal characteristics of memristors

\begin{tabular}{|c|c|c|c|c|}
\hline & 器件 & 优化方法 & 优化性能 & 文献 \\
\hline \multirow{4}{*}{ 导电细丝宽度调节 } & $\mathrm{TiN} / \mathrm{TaO}_{x} / \mathrm{HfAlO}_{x} / \mathrm{TiN}$ & 插人 $\mathrm{TEL}$ 层 $\mathrm{TaO}_{x}$ & Analog特性、电导更新线性 & {$[48,49]$} \\
\hline & $\mathrm{Al} / \mathrm{HfO}_{x} / \mathrm{Ti} / \mathrm{TiN}$ & $\mathrm{A} 1$ 氧化为 $\mathrm{AlO}_{x}$ 阻挡层 & Analog特性、电导更新线性 & {$[50]$} \\
\hline & $\mathrm{TiN} / \mathrm{SiO}_{2} / \mathrm{TaO}_{x} / \mathrm{Pt}$ & 插人 $\mathrm{SiO}_{2}$ 阻挡层 & Analog特性、电导更新线性 & {$[51]$} \\
\hline & TiN/ETML/HfO $/{ }_{x}$ TiN & 插人ETML层 & 电导更新线性 & {$[52]$} \\
\hline \multirow[t]{3}{*}{ 界面势垒调节 } & $\mathrm{Pt}(\mathrm{Al}) / \mathrm{SrTiO}_{3} / \mathrm{Pt}$ & $\mathrm{Al}$ 氧化为 $\mathrm{AlO}_{x}$ 阻挡层 & 保持特性 & {$[53]$} \\
\hline & $\mathrm{Ta} / \mathrm{HfO}_{2} / \mathrm{Pt}$ & 调节限流 & 增加电导态数量 & {$[54]$} \\
\hline & $\mathrm{Pt} / \mathrm{Ta}_{2} \mathrm{O}_{5} / \mathrm{Ru}$ & 采用Ru作为迁移离子 & $\begin{array}{c}\text { 降低能耗 } \\
\text { 保持特性、电导更新线性 }\end{array}$ & {$[55]$} \\
\hline \multirow[t]{4}{*}{ 导电通道成分调节 } & $\mathrm{Ag}-\mathrm{Cu} / \mathrm{Si} / \mathrm{p}$-type $\mathrm{Si}$ & 顶电极采用Ag-Cu合金 & 电导更新线性 & {$[56]$} \\
\hline & $\mathrm{Pd} / \mathrm{Si}: \mathrm{Ta}_{2} \mathrm{O}_{5-x} / \mathrm{TaO}_{y} / \mathrm{Pd}$ & 在阻变层掺杂硅原子 & 电导更新线性、电导更新对称性 & {$[57]$} \\
\hline & $\mathrm{Pt} /-\mathrm{MoO}_{3} / \mathrm{Nb}-\mathrm{SrTiO}_{3}$ & 在- $\mathrm{MoO}_{3}$ 薄膜中注人质子 & 器件均一性 & {$[58]$} \\
\hline & $\mathrm{Pt} / \mathrm{MoO}_{3} / \mathrm{SrCoO}_{2.5} / \mathrm{Nb}-\mathrm{SrTiO}_{3}$ & 增加接受质子的阻变层 & 增加电导态数量、优化器件稳定性 & {$[59]$} \\
\hline
\end{tabular}


增强. 通过调节导电通道成分来调节器件的analog特 性、保持特性、 $I-V$ 线性、电导更新的线性和对称 性等.

\section{2 忆阻突触的仿生特性}

除了需要优化器件的性能外, 还需要考虑忆阻器 对生物突触可塑性的模拟. 突触可塑性包括短时程可 塑性(short-term plasticity，STP)和长时程可塑性(longterm plasticity, LTP). LTP通常可以持续十几分钟以上, 最长可以伴随一生, 而STP的持续就比较短，一般从几 微秒到几分钟. 双脉冲易化(paired pulse facilitation, PPF)和双脉冲抑制(paired pulse depression，PPD)是用 于时间信息处理的典型STP. 当向突触施加成对的脉冲 时，第 2 个脉冲引起的响应电流会比第 1 个脉冲引起的 响应电流更大(更小), 且两个脉冲之间的间隔越大，这 种增强(减弱)效果越小 ${ }^{[60 \sim 64]}$. 我们利用部分易失的器件 $\mathrm{Ag} / \mathrm{Ta}_{2} \mathrm{O}_{5} / \mathrm{CuO} / \mathrm{Pt}$ 实现了 $\mathrm{PPF}^{[65]}$ ，如图3(a)所示; 利用 $\mathrm{WO}_{3}-\mathrm{WSe}_{2}$ 横向异质结模拟了 $\mathrm{STP}$ 和LTP ${ }^{[66]}$, 如图3(c), (d)所示.

生物突触的长时程可塑性分为长时程增强(longterm potentiation, LTP)和长时程抑制(long-term depression, LTD), 当突触接收到脉冲信号时, 其发生LTP或 LTD由接收到脉冲的时序或频率决定，即脉冲时序依 赖可塑性(spike-timing dependent plasticity, STDP)或脉

冲频率依赖可塑性(spike-rating dependent plasticity, SRDP). 实现STDP的方法有很多, 最常见的就是重叠 法, 即向忆阻器的两个端子施加精心设计的脉冲信号, 有效脉冲为两个端子接收到的脉冲之差, 使用设计好 的脉冲可以将时序信息转换为脉冲的幅值信息. 当突 触前脉冲先于突触后脉冲到达时，突触接收到的有效 脉冲幅值的正向部分较大，诱导突触产生LTP; 当突触 前脉冲晚于突触后脉冲到达时，突触接收到的有效脉 冲幅值的负向部分较大, 诱导突触产生LTD; 两个脉冲 到达的间隔时间越长, 有效脉冲的幅值越小, 产生的权 值变化也越小 ${ }^{[68]}$. 重叠法用于实现STDP具有较好的可 靠性, 已经应用于模拟生物的联想学习, 例如使用物理 器件成功模拟了巴甫洛夫条件反射实验 ${ }^{[53]}$.

我们制备了 $\mathrm{Pt} / \mathrm{SrTiO}_{3} / \mathrm{Nb}-\mathrm{SrTiO}_{3}$ 器件，在单个忆阻 器内实现了生物突触的双脉冲(pair)-STDP学习规则. 图3(d)展现了不同初始电导状态下的STDP窗口特性, 表现了经典的赫布(Hebbian)学习特征. 向突触前端 PRE或突触后端POST添加一个脉冲, 即可在该忆阻器 内实现抑制性三脉冲(triplet)-STDP学习规则。测试结 果如图3(e)所示，其由两部分组成，在图中用对角线区 分，曲线的左上角代表人工突触在“2-1”型脉冲 $(2$ 个 PRE脉冲, 1 个POST脉冲)激励作用下的权值变化; 曲线 的右下角代表人工突触在“1-2”型脉冲(1个PRE脉冲，2 个POST脉冲)激励作用下的权值变化. 其中, 红色圆圈

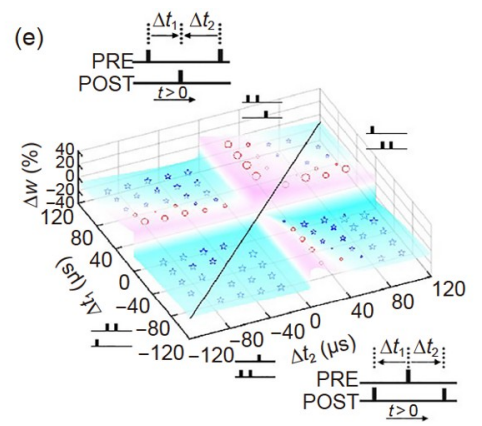

(d)
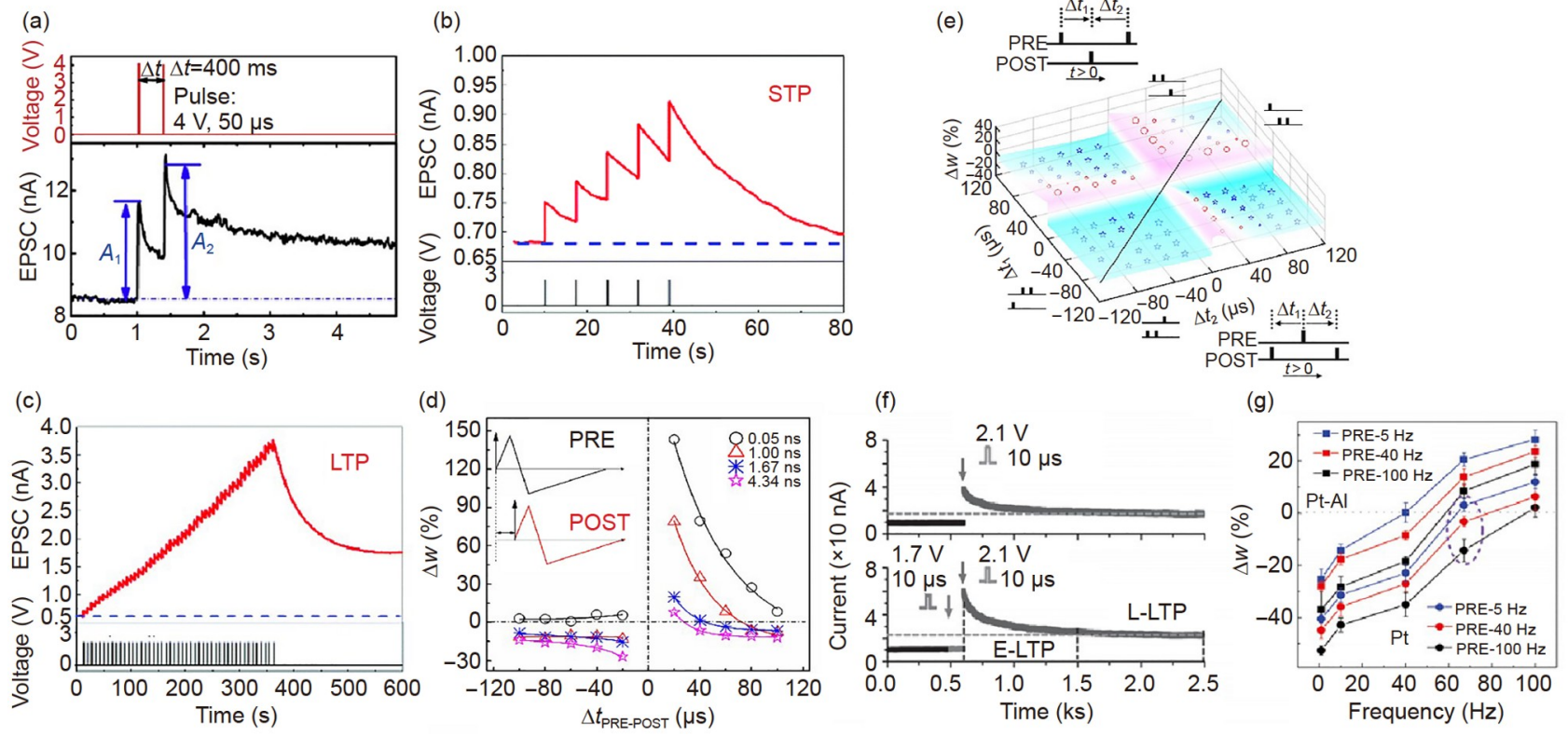

图 3 对突触可塑性功能的模拟. (a) $\mathrm{PPF}^{[65]}$; (b) STP ${ }^{[66]}$; (c) $\mathrm{LTP}^{[66]}$; (d) pair-STDP ${ }^{[67]}$; (e) triplet-STDP ${ }^{[67]}$; (f) 再可塑性 ${ }^{[30]}$; (g) BCM规则 ${ }^{[69]}$ Figure 3 Emulation of the synaptic plasticity. (a) $\operatorname{PPF}^{[65]}$; (b) $\operatorname{STP}^{[66]}$; (c) $\operatorname{LTP}^{[66]}$; (d) pair-STDP ${ }^{[67]}$; (e) triplet-STDP ${ }^{[67]}$; (f) metaplasticity ${ }^{[30]}$; (g) BCM rule $^{[69]}$ 
和蓝色星星代表实验数据，分别表示增强过程和抑制 过程, 标志的大小表示权值相对变化的大小, 背景为根 据Froemke抑制型triplet-STDP数学模型计算得到的理 论权值改变量, 可以发现实验数据与理论预测十分接 近 $^{[67]}$. 抑制性triplet-STDP学习规则的实现统一了突触 的STDP和SRDP学习规则, 为搭建具有时空信息处理 能力的神经网络打下了基础.

此外, 基于忆阻器还实现了更高阶的突触可塑性, 如再可塑性(metaplasticity)和Bienenstock-Cooper-Munro(BCM) 学习规则等. 再可塑性是指突触在权值调节前 接收到的微弱刺激可能会影响权值的改变, 目前已经 报道了几种可以实现再可塑性的忆阻器 ${ }^{[30,69 \sim 71]}$. 以 $\mathrm{Pt} /$ $\mathrm{WO}_{3} / \mathrm{Pt}$ 忆阻器为例(图3(f)), 虽然 $1.7 \mathrm{~V} \times 10 \mu \mathrm{s}$ 的脉冲不 直接改变忆阻突触的权值, 但施加该脉冲后, 该突触对 增强脉冲 $(2.1 \mathrm{~V} \times 10 \mu \mathrm{s})$ 的响应明显变强. $\mathrm{BCM}$ 学习规则 属于SRDP的一种: 突触自身存在 1 个國值, 当突触接收 到的频率大于國值时, 发生LTP; 小于阈值时, 发生LTD; 且该阈值与突触所经历的前期历史有关，当前期接收 的频率过高时，为了保护突触，相应的國值也会被调 高. 我们利用 $\mathrm{Pt}(\mathrm{Al}) / \mathrm{SrTiO}_{3} / \mathrm{Nb}-\mathrm{SrTiO}_{3}$ 忆阻器实现了易失速 度可调的BCM学习规则 ${ }^{[69]}$. 如图3(g)所示, 电极中 $\mathrm{Al}$ 含 量不同的忆阻器表现出不同的阈值调节范围, 通过调 节电极成分, 可以实现突触在不同特征频率下的应用.

\section{3 忆阻神经元}

在神经科学中, 神经元接受来自树突的兴奋性或 抑制性突触电位，脂质双分子层细胞膜的电位则会发 生相应变化. 当整合(integrate)达到一定值时，神经元 则会产生动作电位(action potential, AP), 发出信号 (fire), 并将信号通过轴突经突触传递到下一个神经元, 这是神经元处理和传递信息的过程.

\subsection{Hodgkin-Huxley模型}

为了解释生物神经元的工作模式, 神经科学家霍 奇金(Hodgkin)和赫胥黎(Huxley $)^{[72]}$ 提出了 Hodgkin$\operatorname{Huxley}(\mathrm{HH})$ 模型，HH模型揭示了生物神经元细胞膜上 离子通道(主要是 $\mathrm{Na}^{+}$和 $\mathrm{K}^{+}$通道)的电导率变化和神经元 膜电位之间的关系, 可将 $\mathrm{Na}^{+}$和 $\mathrm{K}^{+}$通道的电位与控制通 道等效为 1 个电池和可调电阻, 将膜电位的被动电学特 性等效为 1 个电容. $\mathrm{HH}$ 模型从基本原理上解释了生物 神经元的工作方式.

目前报道的人工神经元大多都是基于互补金属氧 化物半导体(complementary metal oxide semiconductor, $\mathrm{CMOS}$ ) 器件搭建的 ${ }^{[73 \sim 75]}$, 实现1个神经元的功能需要许 多晶体管或者连接复杂的外围电路，不仅增加了能耗 还降低了集成密度和效率; 与之相比, 忆阻器具有独特 的动态特性, 仅需少量器件或连接简单外围电路即可 实现神经元的功能 ${ }^{[76,77]}$.

美国哈佛机器人实验室(Harvard Robotics Laboratory) $\mathrm{Yi}$ 等人 ${ }^{[78]}$ 利用 2 个 $\mathrm{Pt} / \mathrm{VO}_{2} / \mathrm{Pt}$ 器件分别模拟 $\mathrm{Na}^{+}$和 $\mathrm{K}^{+}$通道. 电容器两端的电压模拟神经细胞膜上的电位, 也称为局部分级电位(local graded potential, LGP), 将 忆阻器和电阻串联后再与电容并联搭建了简单的忆阻 神经元电路(图4(a)), 模拟了神经元中动作电位产生的 过程(图4(b)). 静息状态下, $\mathrm{Na}^{+}$和 $\mathrm{K}^{+}$在浓度梯度的作用 下发生扩散, $\mathrm{Pt} / \mathrm{VO}_{2} / \mathrm{Pt}$ 器件产生约 $0.2 \mathrm{~V}$ 的静息电位. 当 神经元接受到刺激, 前级的电信号导致LGP逐渐升高, 当LGP达到阈值，细胞膜上的 $\mathrm{Na}^{+}$通道和 $\mathrm{K}^{+}$通道先后开 始工作: $\mathrm{Na}^{+}$通道的电导首先增加, 大量 $\mathrm{Na}^{+}$在通道的作 用下进人神经元细胞内, 出现超极化, 膜电位向负向移 动; 当膜电位达到峰值时, $\mathrm{Na}^{+}$通道的电导开始降低, $\mathrm{K}^{+}$通道的电导开始显著增加，大量 $\mathrm{K}^{+}$流出神经元，出 现去极化, 膜电位向正向移动; 随着膜电位的逐渐降 低, $\mathrm{K}^{+}$通道的电导也随之降低, 但是其电导的降低速度 比 $\mathrm{Na}^{+}$通道慢, 导致了过极化电位的出现. 因此, 神经元 动作电位的发放来源于 $\mathrm{K}^{+}$通道和 $\mathrm{Na}^{+}$通道(两个 $\mathrm{Pt} / \mathrm{VO}_{2} /$ Pt器件)的相互配合, 引起LGP的变化.

为了进一步探索忆阻神经元的功能, 本课题组 ${ }^{[43]}$ 制作了一种具有快速易失特性和电池效应的 $\mathrm{W} / \mathrm{WO}_{3} /$ PEDOT:PSS/Pt忆阻器. 具有电池效应的忆阻器内部存 在多种载流子的传输过程，与定制电阻串联后可以模 拟神经元中细胞膜的离子迁移过程. 基于两个 $\mathrm{W} / \mathrm{WO}_{3} /$ PEDOT:PSS/Pt怛器搭建了神经元电路, 包括连接模 块、LGP模块、比较模块和类生物脉冲发放模块, 并 成功实现了类生物脉冲发放和时空信息整合功能. 基 于同样的原理和方式，深圳大学韩素婷课题组 ${ }^{[79]}$ 制作 了 $\mathrm{Au} / \mathrm{MAPbI}_{3} / \mathrm{ITO}$ 忆阻器, 与外围电路一起构建了人 工神经元，成功地模拟了生物神经元的漏电整合发放 行为. 通过修改参数, HH模型可以模拟多种神经元行 为, 但是其动态变量较多, 过于复杂, 不适合用于网络 仿真和大规模集成.

\subsection{IF模型}

Lapicque于1907年提出整合发放(integrate-and-fire, 
(a)

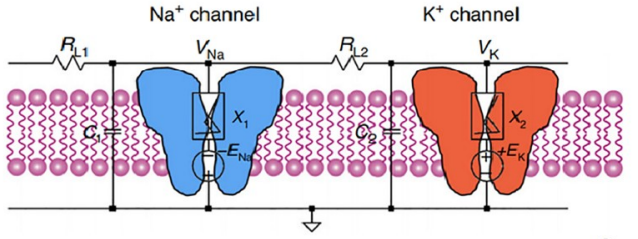

(b)
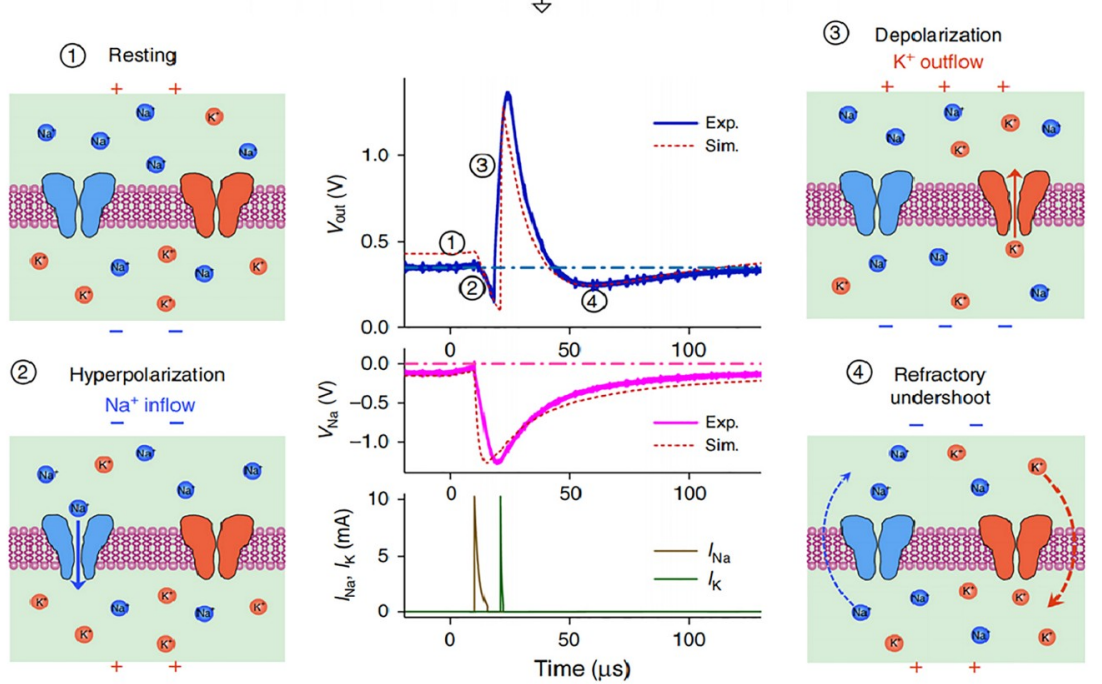

(4) Refractory

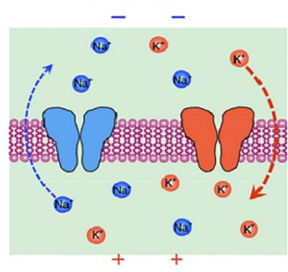

(c)
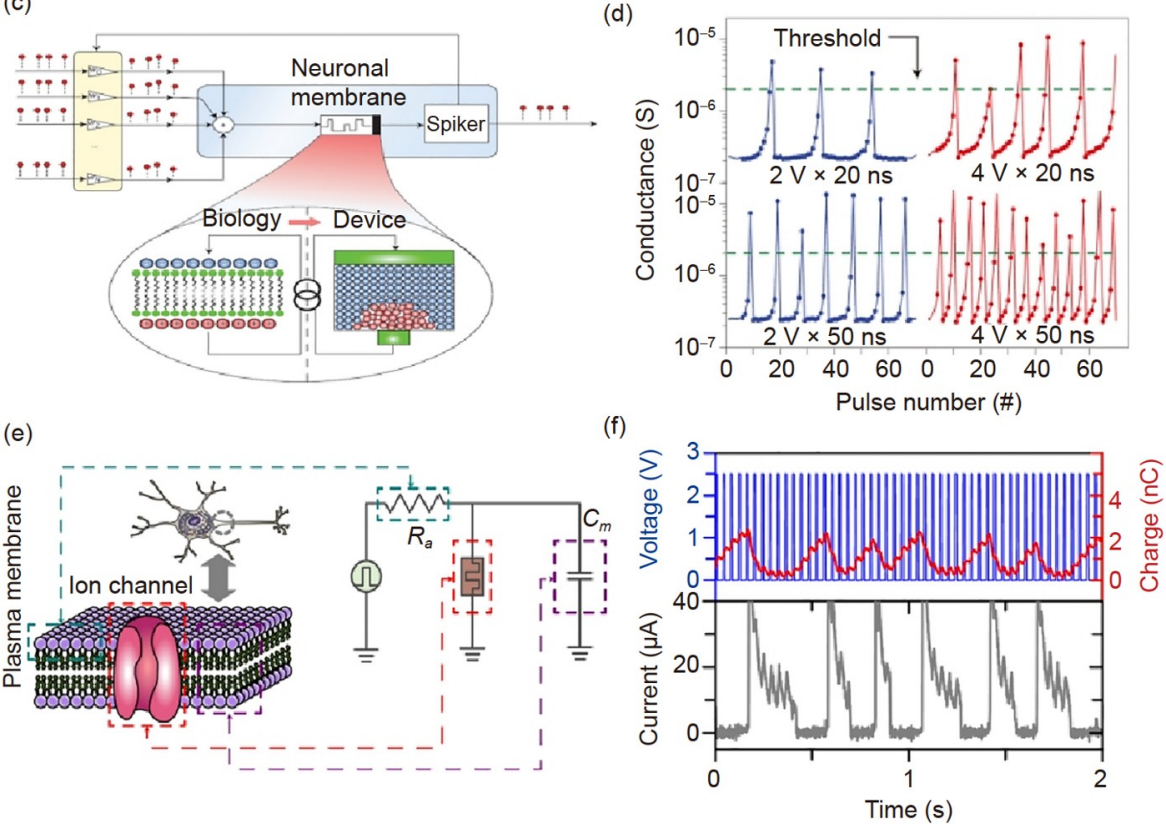

图 4 神经元功能模拟. (a) 基于 2 个Mott器件的神经元电路 ${ }^{[78]}$; (b) 神经元动作电位产生的基本步骤 ${ }^{[78]}$; (c) 基于相变存储器的IF神经元的电路示 意图 ${ }^{[80]}$; (d) 基于相变存储器的IF神经元的电学特性 ${ }^{[80]}$; (e) 基于扩散型忆阻器的LIF神经元的电路结构 ${ }^{[54]}$;(f) 基于扩散型忆阻器的LIF神经元的 电学特性 ${ }^{[54]}$

Figure 4 Emulation of neuronal functions. (a) Circuit of the neuron based Mott devices ${ }^{[78]}$; (b) basic steps in the generation of action potentials (spikes) by a neuron ${ }^{[78]}$; (c) circuit of the IF neuron based on stochastic phase change devices ${ }^{[80]}$; (d) electrical performances of the IF neuron based on stochastic phase change devices ${ }^{[80]}$; (e) circuit of the LIF neuron based on diffusive memristors; (f) electrical behavior of the LIF neuron based on diffusive memristors ${ }^{[54]}$

IF)模型. 该模型忽略了动作电位具体的动态特性, 只考 虑膜电位的被动特性和动作电位的触发, 即将整个神
经元看作一个整体，仅研究神经元的输人和输出关系. 神经元接收到神经脉冲信号后, 膜电位发生变化, 超过 
特定的阈值后开始发放神经脉冲，发放脉冲的形状是 相似的，其传递的信息实质在于某时刻脉冲的有无. Tuma等人 ${ }^{[80]}$ 使用相变忆阻器模拟神经元膜电位 (图4(c)), 实现了具有整合和发放功能的随机神经元. 如 图4(d)所示, 在脉冲的作用下, 器件电导逐渐增加, 直到 超过阈值, 发放脉冲, 然后通过RESET脉冲恢复到初始 状态. 脉冲的幅值或脉宽越大, 器件就会越快发放脉冲, 这与生物特性一致.

IF模型是对HH模型的一种简化，更容易集成于神 经网络中. 但IF模型缺少自发的漏电(leaky)过程, 在没 有神经脉冲时, 膜电位保持不变, 只有完成脉冲发放后, 神经元才将LGP重置到初始状态. 然而实验证明, 具有 leaky功能的神经元模型更具有鲁棒性和泛化性 ${ }^{[81]}$.

\subsection{LIF模型}

漏电整合发放(leaky integrate-and-fire, LIF)模型是 在IF模型的基础上引人了leaky功能，当输人脉冲没有 到达阈值时，膜电位会自发衰减到低于静息电位的状 态, 然后逐渐上升到静息电位, 更具生物合理性. 南加 州大学杨建华课题组 ${ }^{[54]}$ 利用TS 忆阻器与电容并联后再
串联一个电阻组成了LIF神经元(图4(e)), 并联电容上的 电压表示LGP, 当有脉冲时, 电荷在电容中累积, 发生整 合; 当没有脉冲时, 电容发生自发的漏电; 一旦电容电 压高于忆阻器的阈值时, 该器件切换到LRS, 此时电容 的电压无法保持, 开始发放电流脉冲(图4(f)). 为了进一 步降低人工神经元电路的复杂性，华中科技大学缪向 水课题组 ${ }^{[82]}$ 直接利用 $\mathrm{Pt} / \mathrm{Ag} / \mathrm{TiN} / \mathrm{HfAlO}_{x} / \mathrm{Pt}$ 器件与电阻 串联搭建了LIF神经元电路, 模拟了漏电整合发放行为.

研究人员除了利用各种模型实现LIF神经元和类 生物发放功能外, 也致力于探索更多神经元功能的模 拟 ${ }^{[83]}$. 生物神经元在受到刺激产生兴奋时, 会对自身和 相近的神经元产生抑制作用，也就是在一定时间内，即 使再给自身或相近的神经元刺激时，也不会再产生兴 奋. 前者称为不应期(refractory period, RP), 后者称为 侧向抑制(lateral inhibition), 侧向抑制可以避免信息过 载, 支持神经网络进行竞争性学习.

中国科学院微电子研究所刘明课题组 ${ }^{[84]}$ 提出了基 于忆阻器-CMOS混合神经元电路(图5(a)), 其中单个TS 忆阻器可以作为LIF神经元, 两个Dtype锁存器(L1和L2) 和一个与门(G1)用于将发放信号变成固定的脉冲信号.
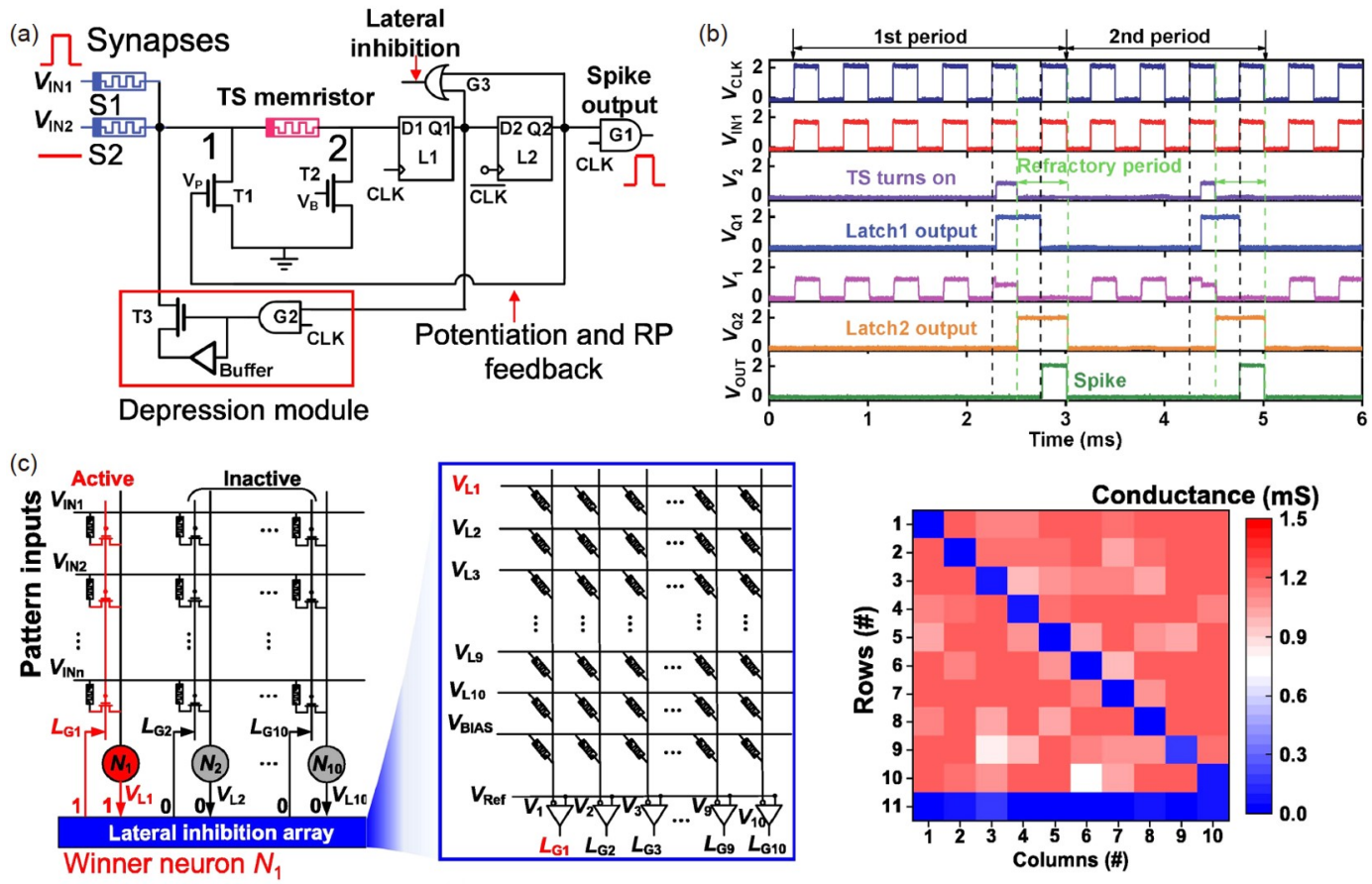

图 5 不应期和侧向抑制功能模拟 ${ }^{[84]}$. (a) 混合神经元的电路图; (b) 当抑制电路关闭时, 连续输人脉冲在两个连续触发周期内的 5 个关键节点输 出值的序列图; (c) 侧向抑制阵列电路原理图和阵列电导态

Figure 5 Emulation of the refractory period and the lateral inhibition ${ }^{[84]}$. (a) Schematic of the hybrid neuron circuit; (b) sequence diagram of the output values of 5 key nodes under continuous input pulses within 2 continuous firing cycles; (c) schematic of the lateral inhibition array circuit and the corresponding conductance states of the array 
其工作过程如图 5(b)所示, 静息状态下，晶体管 $\mathrm{T} 1$ 处于 关断状态, 晶体管 $\mathrm{T} 3$ 栅压为 0 , 低压模块可视为开路. 当 TS 忆阻器被触发时, L2的输出信号作用于 T1的栅极, 使 $\mathrm{T} 1$ 处于 $o n$ 状态, 节点 1 是虚拟的, 为 TS HRS留下一个不应期, 这是通过中断忆阻器的输人来 实现不应期功能的方法. L1和L2的输人信号作用于或 门(G3)产生侧向抑制输出信号, 该信号作用于阵列中 可实现侧向抑制的功能(图5(c)).

\section{4 忆阻神经网络}

将忆阻突触和忆阻神经元连接起来可以搭建忆阻 神经网络, 忆阻神经网络最常见的就是ANN和SNN两 种类型.

\subsection{ANN}

\subsection{1 网络结构}

ANN是对生物神经网络的模拟, 即以数学模型来 模拟生物神经元及突触的结构, 并结合多层次传导来 模拟神经元的互联结构, 是第二代神经网络. 根据网络 的连接方式划分, ANN可分为前馈式神经网络和反馈 式神经网络.

前馈式神经网络的结构包括单层感知器 (single layer perceptron, SLP)、多层感知器(multiple layer perceptron, MLP)和卷积神经网络(convolutional neural net- work, CNN), 结构如图6(a)所示. 其中, SLP和MLP通过 人工突触将相邻两层之间建立联系, 形成全连接(fully connected, FC). SLP仅由输人层和输出层组成, 一般只 能解决线性可分的问题，而MLP添加了额外的隐藏层， 可以用于解决线性不可分的问题. CNN通常由卷积层 (convolutional layer)、池化层(pooling layer)和FC层组 成, 其中卷积层和池化层从输人信息中提取特征, $\mathrm{FC}$ 层 接收已提取的特征并进行推断, $\mathrm{CNN}$ 在图像数据的分 析与处理上颇具优势 ${ }^{[85]}$.

反馈式神经网络中典型的一种是递归神经网络 (recursive neural network), 它是两类人工神经网络的总 称, 分别是时间递归神经网络和结构递归神经网络. 前 者也可称为循环神经网络(recurrent neural network, $\mathrm{RNN}$ )，在传统神经网络的基础上加人了“记忆”成分. RNN的典型结构如图6(b)所示，神经元不仅与上一层 或下一层的神经元连接, 还与同一层的神经元(包括本 身)通过人工突触进行连接，从而在循环中传输信息. 循环的添加使神经网络具备处理前后相关的序列信息 的能力, 因此RNN能够实现数据预测、自然语言处理 和语音识别等功能. 长短程记忆(long short-term memory, LSTM)网络和Hopfield神经网络(Hopfield neural network, HNN)也属于RNN的一种, 只是各层的功能略 有不同. 结构递归神经网络的输人是树/图结构, 这种结 构的标注比较复杂, 一般应用较少.

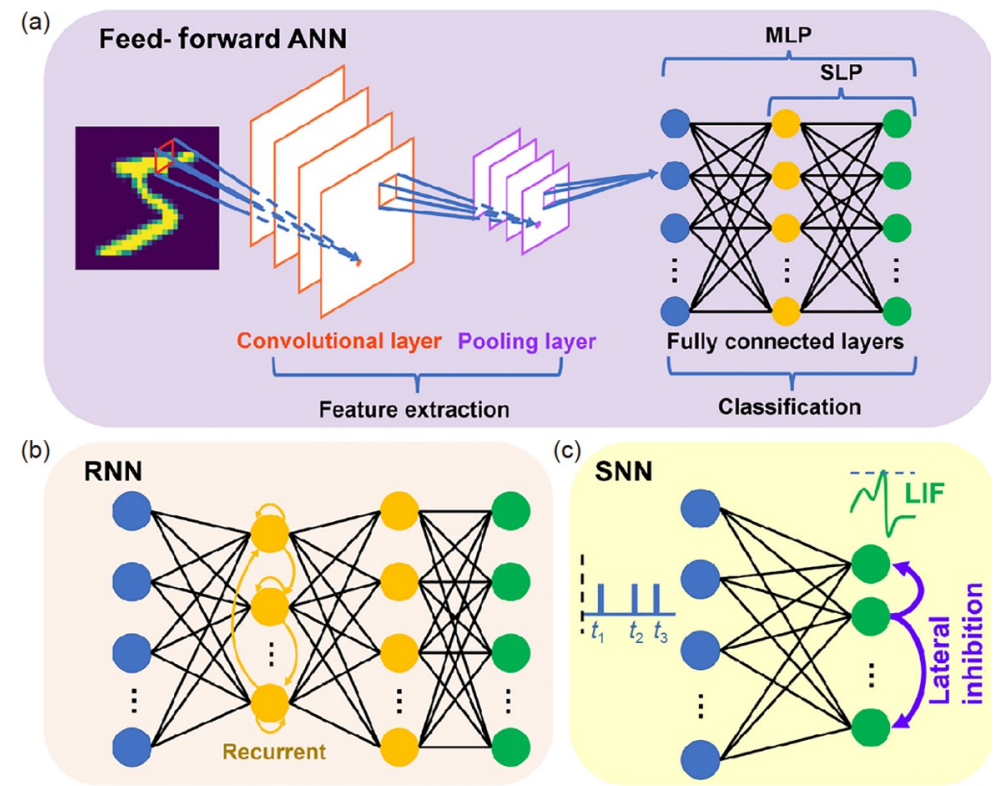

图 6 神经网络的结构 ${ }^{[86]}$. (a) 前馈式神经网络; (b) RNN; (c) SNN

Figure 6 Architectures of neural networks ${ }^{[86]}$. (a) Feed-forward neural network; (b) RNN; (c) SNN 


\subsection{2 学习算法}

ANN中最常用的学习算法是有监督(supervised)的 误差反向传播(error back-propogation)算法, 数据集(dataset)需包括训练集(training set)和测试集(testing set). 训练集中包括输人和期望输出, 进行训练时, 输人由神 经网络处理后得到计算结果，与期望输出比较后得到 误差. 接下来, 将输出层的误差反向传播到每个神经元 层, 以获取单个神经元的误差; 然后, 根据神经元的误 差和输人来计算突触权值所需的改变量. 最后, 对突触 权值进行更新从而完成一次训练. 经多次训练后, 将测 试集的数据输人神经网络中, 检测训练效果.

\subsection{3 硬件实现}

杨建华课题组 ${ }^{[87]}$ 与惠普公司合作, 采用 $\mathrm{Ta} / \mathrm{HfO}_{2} / \mathrm{Pd}$ 结构的忆阻器, 单片集成了 $128 \times 64$ 的 $1 \mathrm{~T} 1 \mathrm{R}(1$ 个晶体管, 1 个忆阻器)阵列(图7(a)). 该阵列有着高达 $99.8 \%$ 的良 率, 实现了信号处理、图像压缩和卷积滤波的功能, 这 些功能在物联网和边缘计算中有重要的应用. 该工作 表明, 在脉宽为 $10 \mathrm{~ns}$ 的读脉冲下, 这个仅仅具有 8192 个 计算单元的芯片的算力能够达到1.64 TOPS(tetra operations per second), 区域面积效率达到1221 TFOPS(tera floating-point operations per second) $/ \mathrm{mm}^{2}$. 与目前设计 的6.28 TFOPS $/ \mathrm{mm}^{2}$ ASIC(application specified integrated chip)芯片相比，效率提高了164倍，证明了基于 忆阻器的神经形态芯片在提升算力方面的巨大潜能. 基于这类器件设计的芯片, 还实现了长/短时程记忆循 环神经网络 ${ }^{[8]}$.
Yao等人 ${ }^{[89]}$ 制作了一个包含 $128 \times 16$ 忆阻器阵列的 处理器(process element, PE)单元(图7(b)), 该阵列表现 出高良率、高性能和高均匀性的优势. 除忆阻器阵列 外，该单元还集成了多个模块，包括输人/输出寄存 器、多路复用器(multiplexer, MUX)、ADC以及用于移 位、加法和控制的模块. 一个PE单元可分为几个小卷 积层的子阵列使用; 多个PE单元也可组合在一起，用 于加速大规模的卷积层或 $F C$ 层. 在定制印制电路板 (printed circuit board, PCB)的帮助下, 8 个PE单元共同 实现了 1 个全硬件的 $\mathrm{CNN}$, 其中 6 个 $\mathrm{PE}$ 单元用于卷积层, 其余PE单元用于 FC层, 最终以极低的能耗实现了 MNIST数据集中手写体数字的识别.

目前，基于忆阻器的ANN已经应用于越来越多的 领域中，如任务系统控制 ${ }^{[00 ~ 92] 、}$ 、特征提取、降维 ${ }^{[50]}$ 和 数据聚类 ${ }^{[93]}$ 等. 但因运算量较大, 在需要海量数据运算 需求的领域，如自动驾驶、机器人或物联网等应用中 会遇到能耗瓶颈.

\section{$4.2 \mathrm{SNN}$}

在神经科学中，视觉、听觉等许多生物神经系统 都采用神经元发放的动作电位(即神经脉冲)的时间来 编码信息, 因此, 更加接近生物神经网络的神经元结构 和运行机理的第三代神经网络模型—SNN应运而生, 它的出现缩小了神经科学和机器学习两者之间的差距. SNN采用精确定时的脉冲序列来编码神经信息，直接 利用神经元的脉冲发放时间作为网络模型的输人与输
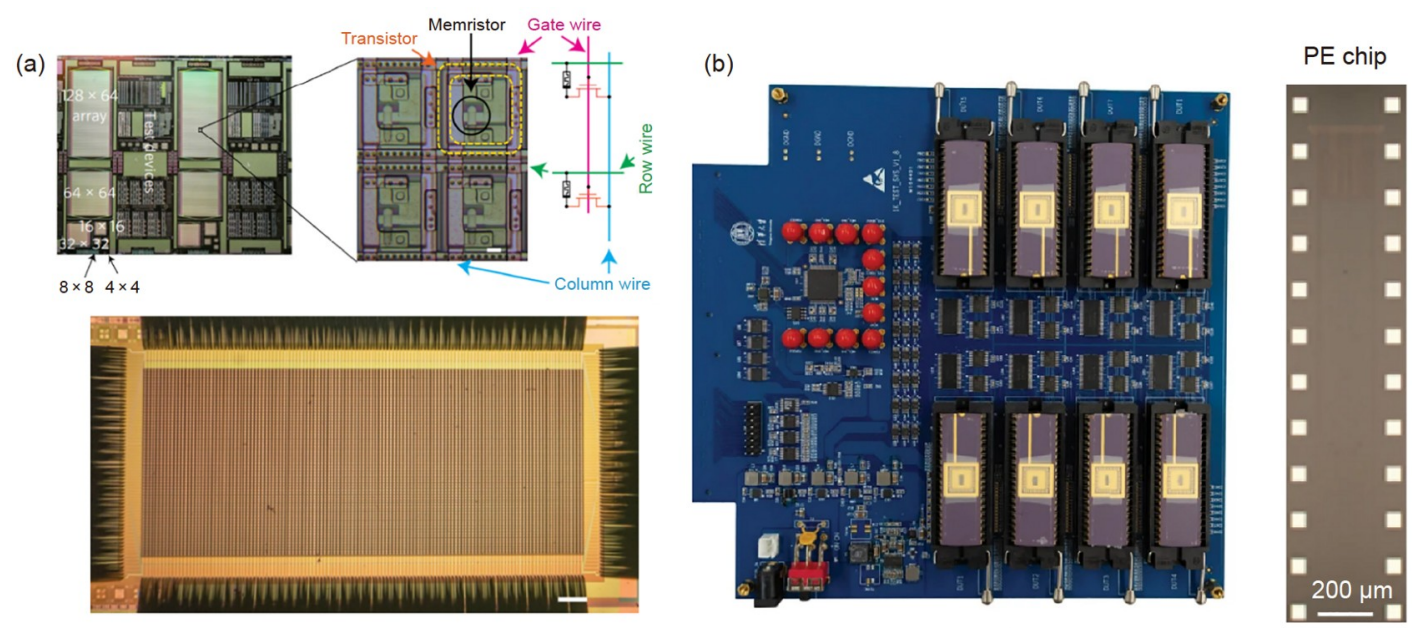

图 7 ANN的硬件实现. (a) $\mathrm{HfO}_{2}$ 基128×64 1T1R阵列(标尺: $\left.500 \mu \mathrm{m}\right)^{[8]}$ 阻器阵列和片上解码器电路组成的部分PE芯片的图像 ${ }^{[89]}$

Figure 7 Hardware implementation of ANNs. (a) $\mathrm{HfO}_{2}$ based $128 \times 64$ 1T1R array (scale bar: $\left.500 \mu \mathrm{m}\right)^{[87]}$. (b) Left: Photograph of the integrated PCB subsystem, also known as the PE board; right: Image of a partial PE chip consisting of a 2048-memristor array and on-chip decoder circuits ${ }^{\text {[89] }}$ 
出, 具有更强大的计算能力, 可实现稀疏和高效的信息 传输、转换和存储, 也可以任意逼近非线性函数和模 拟生物神经网络信号. 更重要的是, 相较于ANN, SNN 是 “事件”驱动型, 具有低能耗、快速推理、在线学 习、大规模并行性和事件驱动信息处理等优点，是处 理复杂时空信息的有效工具.

\subsection{1 网络结构}

SNN 的结构与前馈式 ANN、 RNN类似 ${ }^{[94 ~ 96]}$ (图6(c)), 也分为3种拓扑结构: 前馈脉冲神经网络(feedforward spiking neural network, FSNN)、递归脉冲神经 网络(recurrent spiking neural network, RSNN)和混合型 脉冲神经网络(hybird spiking neural network, HSNN). (1) FSNN. 与FNN不同的是, SNN中 2 个神经元之间可 采用多突触连接，每个突触的权值都是可调的且具有 不同的延时，这延长了突触前神经元输人脉冲对突触 后神经元作用的时间. 另外, 不同的输人脉冲因不同的 突触权值产生突触后电位也不同. (2) RSNN. RSNN是 指网络中具有反馈回路的 SNN, 由于其信息编码及反 馈机制不同于传统递归人工神经网络，由此网络的学 习算法构建及动力学分析较为困难. RSNN可应用于诸 多复杂问题的求解中, 如语言建模、手写数字识别以 及语音识别等. (3) HSNN. HSNN即包括前馈型结构和 递归型结构.

\subsection{2 学习算法}

SNN的学习方式主要包括无监督学习(unsupervised learning)和监督学习(supervised learning)等. (1) 无 监督学习. SNN的无监督学习算法与ANN的类似, 只有 输人信号和实际输出信号, 没有“教师”信号的监督, 靠 网络自身发现学习特征或相关性，自适应性地调整突 触权值, 实现识别、决策和分类等功能, 适合对无标签 数据集进行训练. 此外, SNN可以选用STDP学习规则, 根据突触前神经元和突触后神经元发放的脉冲序列的 相对时序关系对突触权值进行调整. 直接通过硬件实 现神经学中的STDP/SRDP学习规则具有较高的复杂 度, 因此, 研究者提出了一种简化的STDP学习规则: 其 中LTP仅当时间间隔 $<5 \mathrm{~ms}$ 时发生，且突触前脉冲必须 早于突触后脉冲; 其他情况均发生 $L T P^{[97,98]}$. 目前, 除 了学习规则的简化，向输出神经元添加侧向抑制的方 法也得到广泛应用，即在同一个时间段内，只能有 1 个 输出神经元发放脉冲, 该功能也被称为“赢者通吃(winner-take-all, WTA)”. 这增加了STDP/SRDP的适应性, 且 由于输出脉冲发放次数的减少, 进一步降低了系统的

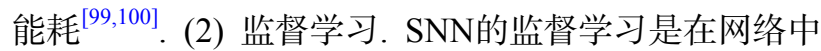
输人多个脉冲序列和目标脉冲序列, 对比实际输出脉 冲序列与目标脉冲序列之间的误差，再根据误差大小 来调整权值. 对于SNN来说, 神经信息以脉冲序列的形 式表示, 神经元内部状态变量及误差函数不再满足连 续可微分的性质，构建有效的SNN监督学习算法非常 困难.

\subsection{3 硬件实现}

因缺乏成熟的训练算法和需要复杂的外围电路, SNN的硬件实现面临巨大的挑战 ${ }^{[4,101 ~ 104]}$. 目前，大多 数研究团队是先通过构建小规模的硬件忆阻神经网络 实现简单的模式识别任务，再通过软件验证大规模 SNN的可行性.

杨建华课题组 ${ }^{[54]}$ 将基于扩散型忆阻器的LIF神经 元与 $8 \times 8$ 忆阻突触阵列集成在同一个芯片上，构建了一 个完整的SNN(图8(a)). 该网络使用STDP学习规则进行 无监督训练，实现了“ $U 、 M 、 A 、 S$ ”四个字母的识别 (图8(b))

深圳大学韩素婷课题组 ${ }^{[79]}$ 将 LIF 神经元与基于 $\mathrm{Ag} /$ $\mathrm{Al}_{2} \mathrm{O}_{3} / \mathrm{Au}$ 的忆阻突触相结合, 搭建了一个简单的 $2 \times 2$ 阵 列SNN. 将突触权值 (W1 W4) 预先设定为合适的值, 不 同时间序列的动作电位通过不同突触权重的突触从前 神经元传递到后神经元，该网络实现了时空模式识别 功能. 刘明课题组 ${ }^{[105]}$ 提出了一种具有 $1 \mathrm{~T} 1 \mathrm{R}$ 结构的神经 元，该神经元的触发速率依赖于栅压，实现了深度学习 网络(deep learning network, DLN)中的ReLU激活功能. 基于该神经元和 $128 \times 64$ 忆阻突触阵列搭建了基于单层 转换的 SNN $(320 \times 10)$ (图8(c)). 利用随机梯度下降算法 提取1T1R神经元膜电位, 对网络进行训练, 在MNIST 数据集分类中，采用1T1R神经元的SNN转换正确率达 到 $85.7 \%$ ，接近仿真神经元的识别正确率 $(86 \%)$. 最后, 借助神经元中的晶体管，提出了一种带有 $1 \mathrm{~T} 1 \mathrm{R}$ 神经 元、外围电路和集成的3D忆阻突触阵列的交叉(crossbar)架构(图8(d))，以实现并行多任务和更好的系统集 成. 在 $3 \mathrm{D}$ 权重数组中, 每一行多层(或多行)执行一个任 务. 在每个推理步骤中, 通过MUX来选择行, 通过 10 个 并行任务进行仿真.

北京大学杨玉超课题组 ${ }^{[106]}$ 制备了一个基于 $\mathrm{NbO}_{x}$ 易失性忆阻器的人工神经元，该神经元具有 4 个关键特 征：阈值驱动的峰值、时空整合、动态逻辑(包括非线 性可分的异或)和增益调制，超越了简单神经元模型所 描述的神经元功能. 该神经元和非易失性 $\mathrm{TaO}_{x}$ 忆阻突 
(a)

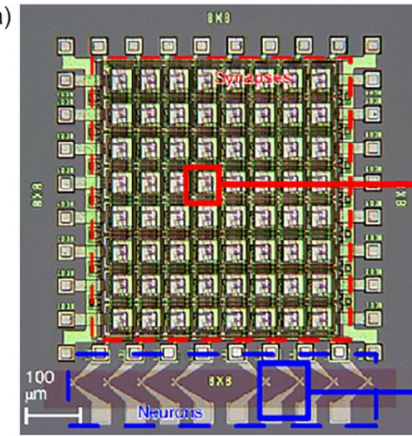

(b)

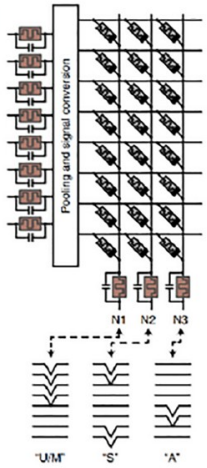

(c)

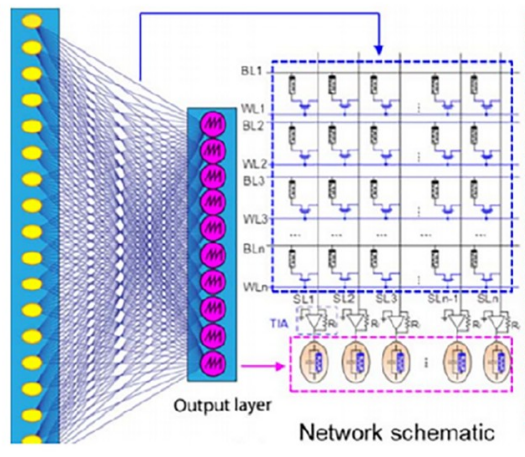

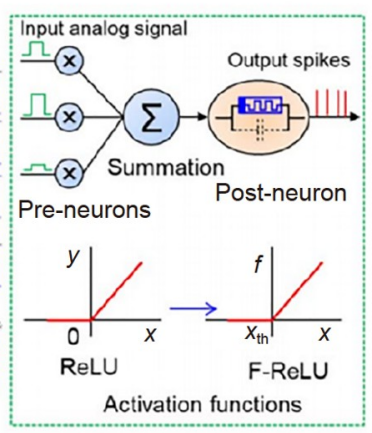

(e)

(f)

(d)

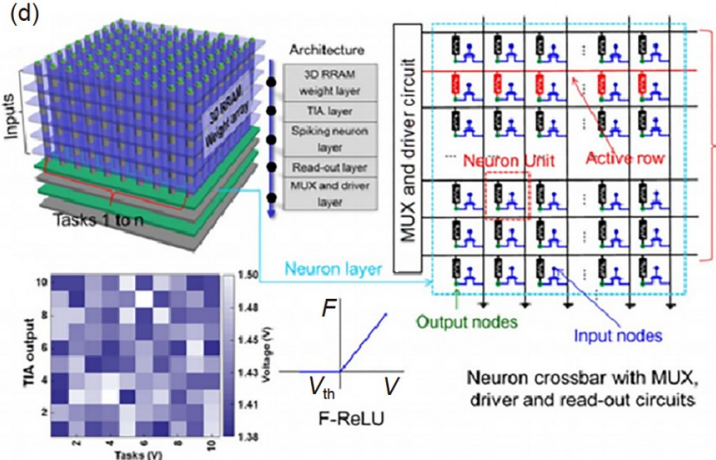

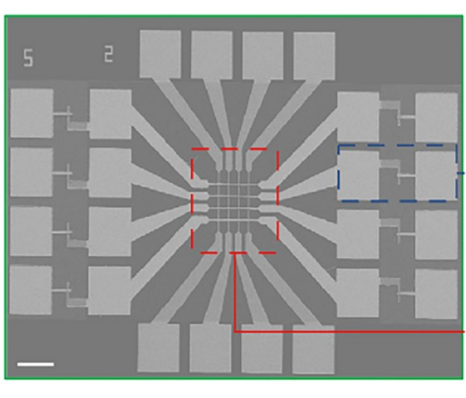

图 $8 \mathrm{SNN}$ 的硬件实现. (a) 由 $8 \times 8$ 1T1R 忆阻突触阵列与 8 个扩散型忆阻人工神经元组成的集成式忆阻神经网络的光学显微图 ${ }^{[54]}$. (b) 基于神经 元输出的 $8 \times 3$ 网络示意图, 训练后神经元的响应分别对应输人字母 $U 、 M 、 A$ 和 $S^{[54]}$. (c) 单层SNN硬件原理图, 以及DLN中的ReLU和F-ReLU功 能 $^{[105]}$. (d) 带有1T1R 神经元、外围电路和集成的3D忆阻突触阵列的crossbar架构 ${ }^{[105]}$. (e) 单片集成忆阻神经网络的扫描电子显微镜图像 ${ }^{[106]}$. (f) 不同输人模式下神经元输出, 其中神经元识别“ 0101 , ”[106]

Figure 8 Hardware implementations of SNNs. (a) Optical micrograph of the integrated memristive neural network, consisting of an $8 \times 81$ T1R memristive synapse crossbar interfacing with 8 diffusive memristive neurons ${ }^{[54]}$. (b) Schematic diagram of an $8 \times 3$ network with inputs based on the outputs of the neurons. The prototypical patterns of the neurons after training correspond to the input letters $U$, M, A, and $\mathrm{S}^{[54]}$. (c) Schematic of onelayer SNN, ReLU function in DLN and F-ReLU activation function in SNN ${ }^{105]}$. (d) Proposed crossbar architecture and its peripheral circuits ${ }^{[105]}$. (e) Scanning electron microscopy (SEM) image of the monolithically integrated memristive neural network ${ }^{[106]}$. (f) Neuronal outputs when presented with different input patterns. Neuron 1 learnt to recognize pattern " 0101, , [106]

触组成了一个基于全忆阻器的 $4 \times 4$ 阵列 $\mathrm{SNN}$ (图 $8(\mathrm{e})$ ), 实验与模拟结果展示了基于简化的 $\operatorname{Delta}(\delta)$ 规则和重合 检测的在线学习进行模式识别的能力. 此外, 人工神经 元的增益调制有助于忆阻神经网络的感受野重映射, 可显著提高人工视觉系统的稳定性. 最后, 为了展示现 有器件在构建大规模全忆阻器 SNN中的潜力, 基于实 验数据通过仿真构建了一个基于 $\mathrm{NbO}_{x}$ 神经元和 $\mathrm{TaO}_{x}$ 突 触的3层全忆阻器SNN(图8(f)). 该网络在MNIST手写体 数字分类中测试准确率达到 $83.24 \%$.

\section{5 总结与展望}

由于集存储与计算于一体的能力和便于实现脑启 发式的神经网络算法的优势, 基于忆阻器的神经形态 计算有着光明的前景. 本文概述了离子型忆阻器在材 料、器件和系统层面所面临的问题, 通过选择合适的
忆阻材料和设计器件结构可以获得所需的器件性能, 并对优化器件非理想特性的方法作了总结; 阐述了忆 阻器作为人工突触和神经元的要求与应用, 并介绍了 忆阻神经网络的结构、学习算法和硬件实现. 虽然忆 阻神经网络在图像、视觉、语音识别等实际应用方面 取得了一些显著成果, 但是就现阶段来说, 忆阻神经网 络还是一个很新的研究领域, 尚处于初步发展阶段, 因 此，其在器件制造、性能优化和学习算法等方面都有 待进一步探索与研究. 目前忆阻神经网络的硬件实现 (如神经形态芯片)和实际应用所面临的挑战主要有以 下几方面.

(1) 器件制造. 现行的半导体CMOS工艺主要服务 于电子器件的制作，如何利用现有的工艺实现忆阻器 中迁移离子(如氧空位等)的调控将是未来忆阻器走向 实用化的挑战; 另一方面, 忆阻器制造会用到一些特殊 
工艺(如高温、转移等), 如何使这些工艺与CMOS工艺 兼容也是未来忆阻器开发需要解决的问题.

(2) 器件阵列化. 只有将器件做成大规模阵列才可 以拓宽其应用场景, 实现更复杂的任务, 但是在扩大阵 列规模的过程中会遇到器件之间相互影响和均一性不 高的问题, 因此解决这些问题还需要大量的研究工作.

(3) 器件能耗. 忆阻神经网络中能耗较大的是外围 电路(特别是ADC) 和突触器件的读操作, 进一步简化外 围电路和选择合适的突触器件材料对减少器件能耗至 关重要.

(4) 神经元模型. 为了使神经元更好地集成于神经 网络中，一种國值可调的简化神经元模型——脉冲响 应模型(spike response model, SRM)应运而生 ${ }^{[107]}$. SRM 模型的工作原理如下: 在脉冲信号的作用下, 膜电位会 上升到超过阈值，输出脉冲信号的同时也会提高阈值， 当再次施加相同的脉冲时，就不会达到阈值而发出输
出脉冲了, 这可以实现神经元的不应期功能; 当相近神 经元被激发后，神经元可以通过提高自身阈值而使自 身不被激发，实现侧向抑制功能. 但是利用硬件构建 SRM模型实现不应期和侧向抑制功能的研究还处于初 始阶段，目前还没有相关报道，因此，基于硬件实现该 模型和更多神经元功能还有待进一步探索.

(5) 学习算法. 算法选择上, 多尝试SNN与ANN结 合的算法, 这样既能利用非易失忆阻器的存算一体功 能, 又能利用忆阻器处理时空信息的高效性, 开发出低 时延、低能耗的人工智能芯片.

作为一种新型的、基于模拟器件的计算方法，忆 阻神经网络需要大量的模数转换和数模转换模块，以 实现忆阻神经网络与现有数字计算的结合. 在这方面 与完全使用模拟计算的生物神经系统还有较大差距, 如何利用忆阻神经网络完成信息获取、处理和响应的 全过程是未来重要的发展方向.

\section{参考文献}

1 Versace M, Chandler B. The brain of a new machine. IEEE Spectr, 2010, 47: 30-37

2 Ielmini D, Wong H S P. In-memory computing with resistive switching devices. Nat Electron, 2018, 1: 333-343

3 Wong H S P, Salahuddin S. Memory leads the way to better computing. Nat Nanotechnol, 2015, 10: 191-194

4 Indiveri G, Liu S C. Memory and information processing in neuromorphic systems. Proc IEEE, 2015, 103: 1379-1397

5 Waser R, Aono M. Nanoionics-based resistive switching memories. Nat Mater, 2007, 6: 833-840

6 Raoux S, Wełnic W, Ielmini D. Phase change materials and their application to nonvolatile memories. Chem Rev, 2010, 110: 240-267

7 Kent A D, Worledge D C. A new spin on magnetic memories. Nat Nanotechnol, 2015, 10: 187-191

8 Mikolajick T, Dehm C, Hartner W, et al. FeRAM technology for high density applications. Microelectron Reliab, 2001, 41: 947-950

9 Strukov D B, Snider G S, Stewart D R, et al. The missing memristor found. Nature, 2008, 453: 80-83

10 Waser R. Redox-based resistive switching memories. J Nanosci Nanotechnol, 2012, 12: 7628-7640

11 Pan R, Li J, Zhuge F, et al. Synaptic devices based on purely electronic memristors. Appl Phys Lett, 2016, 108: 013504

12 Wang J, Pan R, Cao H, et al. Anomalous rectification in a purely electronic memristor. Appl Phys Lett, 2016, 109: 143505

$13 \mathrm{Hu} \mathrm{L}$, Yang J, Wang J, et al. All-optically controlled memristor for optoelectronic neuromorphic computing. Adv Funct Mater, 2021, 31 : 2005582

14 Boybat I, Le Gallo M, Nandakumar S R, et al. Neuromorphic computing with multi-memristive synapses. Nat Commun, 2018, 9: 2514

15 Pantazi A, Woźniak S, Tuma T, et al. All-memristive neuromorphic computing with level-tuned neurons. Nanotechnology, 2016, 27: 355205

16 Kozicki M N, Park M, Mitkova M. Nanoscale memory elements based on solid-state electrolytes. IEEE Trans Nanotechnol, 2005, 4: 331-338

17 Gaba S, Cai F, Zhou J, et al. Ultralow sub-1-nA operating current resistive memory with intrinsic non-linear characteristics. IEEE Electron Device Lett, 2014, 35: 1239-1241

18 Lee J, Lu W D. On-demand reconfiguration of nanomaterials: When electronics meets ionics. Adv Mater, 2018, 30: 1702770

19 Guo X, Schindler C, Menzel S, et al. Understanding the switching-off mechanism in $\mathrm{Ag}^{+}$migration based resistively switching model systems Appl Phys Lett, 2007, 91: 133513

20 Celano U, Goux L, Belmonte A, et al. Three-dimensional observation of the conductive filament in nanoscaled resistive memory devices. Nano Lett, 2014, 14: 2401-2406

21 Choi S, Tan S H, Li Z, et al. SiGe epitaxial memory for neuromorphic computing with reproducible high performance based on engineered dislocations. Nat Mater, 2018, 17: 335-340

22 Miao X S, Li Y, Sun H J, et al. Introduction to Memristor (in Chinese). Beijing: Science Press, 2018 [缪向水, 李祎, 孙华军, 等. 忆阻器导论. 北 京: 科学出版社, 2018]

23 Shao Z Y, Huang H M, Guo X. Optimizing linearity of weight updating in TaO-based memristors by depression pulse scheme for neuromorphic 
computing. Solid State Ion, 2021, 370: 115746

24 Mou X, Tang J, Lyu Y, et al. Analog memristive synapse based on topotactic phase transition for high-performance neuromorphic computing and neural network pruning. Sci Adv, 2021, 7: eabh0648

25 Yang J J, Pickett M D, Li X, et al. Memristive switching mechanism for metal/oxide/metal nanodevices. Nat Nanotechnol, 2008, 3: 429-433

26 Fang Z, Yu H Y, Li X, et al. $\mathrm{HfO}_{x} / \mathrm{TiO}_{x} / \mathrm{HfO}_{x} / \mathrm{TiO}_{x}$ multilayer-based forming-free RRAM devices with excellent uniformity. IEEE Electron Device Lett, 2011, 32: 566-568

27 Lee M J, Lee C B, Lee D, et al. A fast, high-endurance and scalable non-volatile memory device made from asymmetric $\mathrm{Ta}_{2} \mathrm{O}_{5-x} / \mathrm{TaO}_{2-x} \mathrm{bilayer}$ structures. Nat Mater, 2011, 10: 625-630

28 Lee W, Park J, Kim S, et al. High current density and nonlinearity combination of selection device based on $\mathrm{TaO}_{x} / \mathrm{TiO}_{2} / \mathrm{TaO}_{x}$ structure for one selector-one resistor arrays. ACS Nano, 2012, 6: 8166-8172

29 Yoon J H, Kim K M, Song S J, et al. $\mathrm{Pt} / \mathrm{Ta}_{2} \mathrm{O}_{5} / \mathrm{HfO}_{2-x} / \mathrm{Ti}$ resistive switching memory competing with multilevel NAND flash. Adv Mater, 2015, 27: $3811-3816$

30 Tan Z H, Yang R, Terabe K, et al. Synaptic metaplasticity realized in oxide memristive devices. Adv Mater, 2016, 28: 377-384

31 Govoreanu B, Kar G S, Chen Y Y, et al. $10 \times 10 \mathrm{~nm}^{2} \mathrm{Hf} / \mathrm{HfO}_{x}$ crossbar resistive RAM with excellent performance, reliability and low-energy operation. In: IEEE International Electron Devices Meeting (IEDM). IEEE: Washington DC, 2011

32 Pan F, Gao S, Chen C, et al. Recent progress in resistive random access memories: Materials, switching mechanisms, and performance. Mater Sci Eng-R-Rep, 2014, 83: 1-59

33 Baeumer C, Schmitz C, Ramadan A H H, et al. Spectromicroscopic insights for rational design of redox-based memristive devices. Nat Commun, 2015, 6: 8610

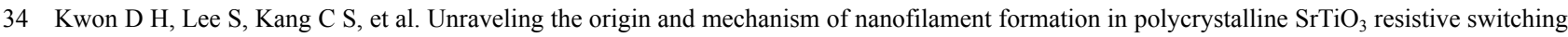
memories. Adv Mater, 2019, 31: 1901322

35 Mikheev E, Hoskins B D, Strukov D B, et al. Resistive switching and its suppression in Pt/Nb:SrTiO 3 junctions. Nat Commun, 2014, 5: 3990

36 Yang R, Li X M, Yu W D, et al. Endurance improvement of resistance switching behaviors in the $\mathrm{La}_{0.7} \mathrm{Ca}_{0.3} \mathrm{MnO}_{3}$ film based devices with $\mathrm{Ag}$ - $\mathrm{Al}$ alloy top electrodes. J Appl Phys, 2010, 107: 063703

37 Yang R, Li X. Improvement of resistance switching properties for metal/ $\mathrm{La}_{0.7} \mathrm{Ca}_{0.3} \mathrm{MnO}_{3} / \mathrm{Pt}$ devices. Phys Status Solidi A-Appl Mat, 2011, 208: $1041-1046$

38 Choi B J, Torrezan A C, Strachan J P, et al. High-speed and low-energy nitride memristors. Adv Funct Mater, 2016, 26: 5290-5296

39 Chen W, Gao S, Yi X, et al. Organic and hybrid resistive switching materials and devices. J Funct Polym, 2019, 32: 434-447

40 Zhang B, Fan F, Xue W, et al. Redox gated polymer memristive processing memory unit. Nat Commun, 2019, 10: 736

41 Zhang C, Ye W B, Zhou K, et al. Bioinspired artificial sensory nerve based on nafion memristor. Adv Funct Mater, 2019, 29: 1808783

42 Menzel S, Waters M, Marchewka A, et al. Origin of the ultra-nonlinear switching kinetics in oxide-based resistive switches. Adv Funct Mater, 2011, 21: 4487-4492

43 Huang H M, Yang R, Tan Z H, et al. Quasi-Hodgkin-Huxley neurons with leaky integrate-and-fire functions physically realized with memristive devices. Adv Mater, 2019, 31: 1803849

44 Wang Z, Rao M, Han J W, et al. Capacitive neural network with neuro-transistors. Nat Commun, 2018, 9: 3208

45 Zhang W, Gao B, Tang J, et al. Neuro-inspired computing chips. Nat Electron, 2020, 3: 371-382

46 Olshausen B A. Theoretical neuroscience: Computational and mathematical modeling of neural systems. J Cogn Neurosci, 2003, 15: 154-155

47 Tang J, Yuan F, Shen X, et al. Bridging biological and artificial neural networks with emerging neuromorphic devices: Fundamentals, progress, and challenges. Adv Mater, 2019, 31: 1902761

48 Yao P, Wu H, Gao B, et al. Face classification using electronic synapses. Nat Commun, 2017, 8: 15199

$49 \mathrm{Wu} \mathrm{W}, \mathrm{Wu} \mathrm{H}, \mathrm{Gao} \mathrm{B}$, et al. Improving analog switching in $\mathrm{HfO}_{x}$-based resistive memory with a thermal enhanced layer. IEEE Electron Device Lett, 2017, 38: 1019-1022

50 Woo J, Moon K, Song J, et al. Improved synaptic behavior under identical pulses using $\mathrm{AlO}_{x} / \mathrm{HfO}_{2}$ bilayer RRAM array for neuromorphic systems. IEEE Electron Device Lett, 2016, 37: 994-997

51 Wang Z, Yin M, Zhang T, et al. Engineering incremental resistive switching in $\mathrm{TaO}_{x}$ based memristors for brain-inspired computing. Nanoscale, 2016, 8: 14015-14022

$52 \mathrm{Wu} \mathrm{W}, \mathrm{Wu} \mathrm{H}, \mathrm{Gao}$ B, et al. A methodology to improve linearity of analog RRAM for neuromorphic computing. In: 2018 IEEE Symposium on VLSI Technology. New York: IEEE, 2018. 103-104

53 Tan Z H, Yin X B, Yang R, et al. Pavlovian conditioning demonstrated with neuromorphic memristive devices. Sci Rep, 2017, 7: 713

54 Wang Z, Joshi S, Savel'ev S, et al. Fully memristive neural networks for pattern classification with unsupervised learning. Nat Electron, 2018, 1: 137-145 
55 Yoon J H, Zhang J, Lin P, et al. A low-current and analog memristor with Ru as mobile species. Adv Mater, 2020, 32: 1904599

56 Yeon H, Lin P, Choi C, et al. Alloying conducting channels for reliable neuromorphic computing. Nat Nanotechnol, 2020, 15: 574-579

57 Kim S, Choi S H, Lee J, et al. Tuning resistive switching characteristics of tantalum oxide memristors through Si doping. ACS Nano, 2014, 8: 10262-10269

58 Wang Z, Yang R, Huang H M, et al. Electroforming-free artificial synapses based on proton conduction in $\alpha$-MoO $\mathrm{O}_{3}$ films. Adv Electron Mater, 2020, 6: 1901290

59 Wang Z, Huang H M, Guo X. Memristive devices with multiple resistance states based on the migration of protons in $\alpha-\mathrm{MoO}_{3} / \mathrm{SrCoO}_{2.5}$ stacks. Adv Electron Mater, 2021, 7: 2001243

60 Wang Z, Joshi S, Savelév S E, et al. Memristors with diffusive dynamics as synaptic emulators for neuromorphic computing. Nat Mater, 2017, 16: $101-108$

61 Yang C S, Shang D S, Liu N, et al. A synaptic transistor based on quasi-2D molybdenum oxide. Adv Mater, 2017, 29: 1700906

62 Zhu L Q, Wan C J, Guo L Q, et al. Artificial synapse network on inorganic proton conductor for neuromorphic systems. Nat Commun, 2014, 5: 3158

63 Liu Y H, Zhu L Q, Feng P, et al. Freestanding artificial synapses based on laterally proton-coupled transistors on chitosan membranes. Adv Mater, 2015, 27: 5599-5604

64 Wan C J, Zhu L Q, Liu Y H, et al. Proton-conducting graphene oxide-coupled neuron transistors for brain-inspired cognitive systems. Adv Mater, 2016, 28: 3557-3563

65 Jiang L. Memristive devices based on random mixtures of metals and oxides (in Chinese). Master Thesis. Wuhan: Huazhong University of Science and Technology, 2018 [江莉. 基于金属-氧化物随机混合材料的忆阻器件的研究. 硕士学位论文. 武汉: 华中科技大学, 2018]

$66 \mathrm{He} \mathrm{H} \mathrm{K}$, Yang R, Huang H M, et al. Multi-gate memristive synapses realized with the lateral heterostructure of $2 \mathrm{D} \mathrm{WSe}_{2}$ and $\mathrm{WO}_{3}$. Nanoscale, 2020, 12: 380-387

67 Yang R, Huang H M, Hong Q H, et al. Synaptic suppression triplet-STDP learning rule realized in second-order memristors. Adv Funct Mater, 2018, 28: 1704455

68 Li Y, Zhong Y, Xu L, et al. Ultrafast synaptic events in a chalcogenide memristor. Sci Rep, 2013, 3: 1619

69 Wu Q, Wang H, Luo Q, et al. Full imitation of synaptic metaplasticity based on memristor devices. Nanoscale, 2018, 10: 5875-5881

$70 \mathrm{Li} \mathrm{J}$, Yang Y, Yin M, et al. Electrochemical and thermodynamic processes of metal nanoclusters enabled biorealistic synapses and leaky-integrateand-fire neurons. Mater Horiz, 2020, 7: 71-81

71 Zhu X, Du C, Jeong Y J, et al. Emulation of synaptic metaplasticity in memristors. Nanoscale, 2017, 9: 45-51

72 Hodgkin A L, Huxley A F. A quantitative description of membrane current and its application to conduction and excitation in nerve. J Physiol, 1952, 117: 500-544

73 Indiveri G, Linares-Barranco B, Hamilton T J, et al. Neuromorphic silicon neuron circuits. Front Neurosci, 2011, 5: 73

74 Wijekoon J H B, Dudek P. Compact silicon neuron circuit with spiking and bursting behaviour. Neural Netw, 2008, 21: 524-534

75 Mahowald M, Douglas R. A silicon neuron. Nature, 1991, 354: 515-518

76 Lin J, Annadi A, Sonde S, et al. Low-voltage artificial neuron using feedback engineered insulator-to-metal-transition devices. In: 2016 IEEE Interntional Electron Devices Meeting (IEDM). New York: IEEE, 2016. 34.5

77 Jaiswal A, Roy S, Srinivasan G, et al. Proposal for a leaky-integrate-fire spiking neuron based on magnetoelectric switching of ferromagnets IEEE Trans Electron Devices, 2017, 64: 1818-1824

78 Yi W, Tsang K K, Lam S K, et al. Biological plausibility and stochasticity in scalable $\mathrm{VO}_{2}$ active memristor neurons. Nat Commun, 2018, 9: 4661

79 Yang J Q, Wang R, Wang Z P, et al. Leaky integrate-and-fire neurons based on perovskite memristor for spiking neural networks. Nano Energy, 2020, 74: 104828

80 Tuma T, Pantazi A, Le Gallo M, et al. Stochastic phase-change neurons. Nat Nanotechnol, 2016, 11: 693-699

81 Chowdhury S S, Lee C, Roy K. Towards understanding the effect of leak in spiking neural networks. Neurocomputing, 2021, 464: 83-94

$82 \mathrm{Lu} \mathrm{Y} \mathrm{F,} \mathrm{Li} \mathrm{Y,} \mathrm{Li} \mathrm{H,} \mathrm{et} \mathrm{al.} \mathrm{Low-power} \mathrm{artificial} \mathrm{neurons} \mathrm{based} \mathrm{on} \mathrm{Ag} / \mathrm{TiN} / \mathrm{HfAlO}_{x} / \mathrm{Pt}$ threshold switching memristor for neuromorphic computing. IEEE Electron Device Lett, 2020, 41: 1245-1248

83 Wei Q M, Tang J S, Li X Y, et al. Artificial neuron with spike frequency adaptation based on Mott memristor. In: 2021 5th IEEE Election Devices Technology \& Manufacturing Conference (EDTM). New York: IEEE, 2021, doi: 10.1109/EDTM50988.2021.9421014

84 Zhang X, Lu J, Wang Z, et al. Hybrid memristor-CMOS neurons for in-situ learning in fully hardware memristive spiking neural networks. Sci Bull, 2021, 66: 1624-1633

85 LeCun Y, Bengio Y, Hinton G. Deep learning. Nature, 2015, 521: 436-444

86 Huang H M, Wang Z, Wang T, et al. Artificial neural networks based on memristive devices: From device to system. Adv Intell Syst, 2020, 2: 2000149 
$87 \mathrm{Li} \mathrm{C}, \mathrm{Hu} \mathrm{M}, \mathrm{Li} \mathrm{Y}$, et al. Analogue signal and image processing with large memristor crossbars. Nat Electron, 2018, 1: 52-59

88 Li C, Wang Z, Rao M, et al. Long short-term memory networks in memristor crossbar arrays. Nat Mach Intell, 2019, 1: 49-57

89 Yao P, Wu H, Gao B, et al. Fully hardware-implemented memristor convolutional neural network. Nature, 2020, 577: 641-646

90 Wang C, Yang Z, Wang S, et al. A braitenberg vehicle based on memristive neuromorphic circuits. Adv Intell Syst, 2020, 2: 1900103

91 Wang Z, Li C, Song W, et al. Reinforcement learning with analogue memristor arrays. Nat Electron, 2019, 2: 115-124

92 Jeong Y J, Lee J, Moon J, et al. $K$-means data clustering with memristor networks. Nano Lett, 2018, 18: 4447-4453

93 Choi S, Shin J H, Lee J, et al. Experimental demonstration of feature extraction and dimensionality reduction using memristor networks. Nano Lett, 2017, 17: 3113-3118

94 Zhang A, Zhou H, Li X, et al. Fast and robust learning in spiking feed-forward neural networks based on intrinsic plasticity mechanism. Neurocomputing, 2019, 365: 102-112

95 Demin V, Nekhaev D. Recurrent spiking neural network learning based on a competitive maximization of neuronal activity. Front Neuroinformatics, 2018, 12: 79

96 Kang T S, Banerjee A. Learning feedforward and recurrent deterministic spiking neuron network feedback controllers. 2017, arXiv: 1708.02603

97 Serb A, Bill J, Khiat A, et al. Unsupervised learning in probabilistic neural networks with multi-state metal-oxide memristive synapses. Nat Commun, 2016, 7: 12611

98 Ambrogio S, Balatti S, Milo V, et al. Neuromorphic learning and recognition with one-transistor-one-resistor synapses and bistable metal oxide RRAM. IEEE Trans Electron Devices, 2016, 63: 1508-1515

99 Jiang Y, Huang P, Zhu D, et al. Design and hardware implementation of neuromorphic systems with RRAM synapses and threshold-controlled neurons for pattern recognition. IEEE Trans Circuits Syst I-Regul Pap, 2018, 65: 2726-2738

100 Guo Y, Wu H, Gao B, et al. Unsupervised learning on resistive memory array based spiking neural networks. Front Neurosci, 2019, 13: 812

101 Prezioso M, Mahmoodi M R, Bayat F M, et al. Spike-timing-dependent plasticity learning of coincidence detection with passively integrated memristive circuits. Nat Commun, 2018, 9: 5311

102 Mostafa H, Khiat A, Serb A, et al. Implementation of a spike-based perceptron learning rule using $\mathrm{TiO}_{2-x}$ memristors. Front Neurosci, 2015, 9 : 357

103 Xu Z, Skorheim S, Tu M, et al. Improving efficiency in sparse learning with the feedforward inhibitory motif. Neurocomputing, 2017, 267: 141151

104 Wang W, Pedretti G, Milo V, et al. Learning of spatiotemporal patterns in a spiking neural network with resistive switching synapses. Sci Adv, 2018, 4: eaat 4752

105 Zhang X M, Wang Z R, Song W H, et al. Experimental demonstration of conversion-based SNNs with 1T1R Mott neurons for neuromorphic inference. In: 2019 IEEE International Electron Devices Meeting (IEDM). New York: IEEE, 2019, doi: 10.1109/IEDM19573.2019.8993519

106 Duan Q, Jing Z, Zou X, et al. Spiking neurons with spatiotemporal dynamics and gain modulation for monolithically integrated memristive neural networks. Nat Commun, 2020, 11: 3399

107 Abbott L F. Lapicque's introduction of the integrate-and-fire model neuron (1907). Brain Res Bull, 1999, 50: 303-304 


\title{
Neuromorphic systems based on ionic memristors: From materials, devices to chips
}

\author{
Juan Wen, He-Ming Huang, Zhe Wang \& Xin Guo* \\ State Key Laboratory of Material Processing and Die \& Mould Technology, School of Materials Science and Engineering, Huazhong University of \\ Science and Technology, Wuhan 430074, China \\ * Corresponding author, E-mail: xguo@hust.edu.cn
}

Human society is now moving from informatization to intelligence, in which artificial intelligence (AI) is the key. In many fields, AI has demonstrated the ability to surpass the human brain, due to the continuous innovation of AI algorithms and the rapidly increasing computing powers of super-computers. Considering the current data volume, it is particularly important to improve the computing power. In this aspect, innovative AI chips are critical.

In the pursuit of high performance and low power consumption, AI chips face two bottlenecks. (1) The memory wall: The access speed of memories cannot keep up with the speed of data consuming by computing units, which perplexes the computer architecture for a long time. (2) The failure of Moore's law, is due to the limitation of basic physical principles. It becomes more and more difficult to improve the integration density and reduce the power consumption of chips by reducing the transistor size.

A memristor is a kind of circuit device with memory effect. Under the action of external electric fields, the device can change reversibly between high and low conductance states. The different conductance states are controlled by the migration of various ionic defects, e.g., oxygen vacancies, proton defects, cations, etc. Memristors have the characteristics of simple sandwich structure, fast operation speed, low energy consumption, and rich performances. In addition, memristor arrays with crossbar structure can achieve the integration of the data storage and computing by the vector-matrix multiplication. Therefore, memristor-based neuromorphic computing, with its ability to perform computation where data are generated and the advantages of convenient realization of brain-inspired algorithms, is very promising to crack the memory wall and the failure of Moore's law. Ionic defects are electrically charged defects with a mass that is typically 3 orders of magnitude larger than the mass of an electron. Therefore the ionic transport is not limited by the tunneling effect, so that the Moore's law can be extended to sub-nanometer scale for ionic devices. Frequent data transports between the processing units and the memories are energy- and time-consuming. To overcome such a memory wall, memristors are introduced into the current computer architecture to eliminate the distance between the processing units and the memories. Such a so-called in-memory computing based on memristor is largely inspired by the neurological structure of the biological brain, in which information processing and storage occur in one place.

In this article, recent progress in memristor-related research is reviewed. By carefully selecting transition metal oxides and mobile ions, materials with different ionic mobility are produced. By proper device structure design, memristors with non-volatile, partially volatile and fast volatile characteristics are realized. According to the switching mechanisms, memristors can be classified into several types, and the performance requirements and optimization measures for memristors based on the ionic conduction are discussed. By means of ionic memristors, memristive synapses successfully realize the synaptic plasticity, including the spike-timing dependent plasticity (STDP), the spike-rating dependent plasticity (SRDP), the metaplasticity, and the Bienenstock-Cooper-Munro rule. Moreover, neuron functions are realized based on different neuronal models (the Hodgkin-Huxley (HH) model, the integrate-and-fire (IF) model and the leaky integrate-andfire (LIF) model). Furthermore, memristive neural networks can be realized by connecting the memristive synapses with the memristive neurons. The most common types of memristive neural networks are the artificial neural network (ANN) and the spiking neural network (SNN). The structures, learning algorithms and hardware implementations of ANN and SNN are summarized. Finally, the challenges and prospects of memristive neural networks and neuromorphic chips are elaborated.

memristor, memristive synapse, memristive neuron, memristive neural network, neuromorphic chip

doi: 10.1360/TB-2021-1072 